\title{
Contracts and Technology Adoption
}

\section{Citation}

Acemoglu, Daron, Pol Antras, and Elhanan Helpman. 2007. Contracts and technology adoption. American Economic Review 97(3): 916-943.

\section{Published Version}

http://dx.doi.org/10.1257/aer.97.3.916

\section{Permanent link}

http://nrs.harvard.edu/urn-3:HUL.InstRepos:3199063

\section{Terms of Use}

This article was downloaded from Harvard University's DASH repository, and is made available under the terms and conditions applicable to Other Posted Material, as set forth at http:// nrs.harvard.edu/urn-3:HUL.InstRepos:dash.current.terms-of-use\#LAA

\section{Share Your Story}

The Harvard community has made this article openly available.

Please share how this access benefits you. Submit a story.

Accessibility 


\title{
Contracts and Technology Adoption*
}

\author{
Daron Acemoglu \\ Pol Antràs \\ Department of Economics, MIT Department of Economics, Harvard University \\ Elhanan Helpman \\ Department of Economics, Harvard University
}

November 28, 2006

\begin{abstract}
We develop a tractable framework for the analysis of the relationship between contractual incompleteness, technological complementarities, and technology adoption. In our model a firm chooses its technology and investment levels in contractible activities by suppliers of intermediate inputs. Suppliers then choose investments in noncontractible activities, anticipating payoffs from an ex post bargaining game. We show that greater contractual incompleteness leads to the adoption of less advanced technologies and that the impact of contractual incompleteness is more pronounced when there is greater complementary among the intermediate inputs. We study a number of applications of the main framework and show that the mechanism proposed in the paper can generate sizable productivity differences across countries with different contracting institutions and that differences in contracting institutions lead to endogenous comparative advantage differences.
\end{abstract}

Keywords: comparative advantage, economic growth, incomplete contracts, technology choice, theory of the firm.

JEL Classification: D23, F10, L23, O30.

\footnotetext{
*A previous version of this paper was circulated under the title "Contracts and the Division of Labor." We thank Gene Grossman, Oliver Hart, Kalina Manova, Damián Migueles, Giacomo Ponzetto, Richard Rogerson, Rani Spiegler, two anonymous referees, and participants in the Canadian Institute for Advanced Research and Minnesota Workshop in Macroeconomic Theory conferences, and seminar participants at Harvard, MIT, Tel Aviv, Universitat Pompeu Fabra, Houston, UCSD, Southern Methodist, Stockholm School of Economics, Stockholm University (IIES), ECARES, Colorado-Boulder, Kellogg, NYU, UBC, Georgetown, World Bank, Montreal, Brandeis, Wisconsin, UC Berkeley, Haifa, Syracuse, and Autònoma-Barcelona for useful comments. We also thank Davin Chor, Alexandre Debs and Ran Melamed for excellent research assistance. Acemoglu and Helpman thank the National Science Foundation for financial support. Much of Helpman's work for this paper was done when he was Sackler Visiting Professor at Tel Aviv University.
} 


\section{Introduction}

There is widespread agreement that differences in technology are a major source of productivity differences across firms, industries and nations. ${ }^{1}$ Despite this widespread agreement, we are far from an established framework for the analysis of technology choices of firms. In this paper, we take a step in this direction and develop a simple model to study the impact of contracting institutions, which regulate the relationship between the firm and its suppliers, on technology choices.

Our model combines two well-established approaches. The first is the representation of technology as the range of intermediate inputs used by firms; a greater range of intermediate inputs increases productivity by allowing greater specialization and thus corresponds to more "advanced" technology. ${ }^{2}$ The second is Grossman and Hart's (1986) and Hart and Moore's (1990) approach to incomplete-contracting models of the firm. We study technology choice of firms under incomplete contracts, and extend Hart and Moore's framework, by allowing contracts to be partially incomplete. This combination enables us to investigate how the degree of contractual incompleteness and the extent of technological complementarities between intermediate inputs affect the choice of technology.

In our baseline model a firm decides on technology (on the range of specialized intermediate goods), recognizing that a more advanced technology is more productive, but also entails a variety of costs. In addition to the direct pecuniary costs of engaging more suppliers (corresponding to the greater range of intermediate inputs), a more advanced technology necessitates contracting with more suppliers. All of the activities that suppliers undertake are relationship-specific, and a fraction of those is ex ante contractible, while the rest, as in the work by Grossman-Hart-Moore, are nonverifiable and noncontractible. The fraction of contractible activities is our measure of the quality of contracting institutions. ${ }^{3}$ Suppliers are contractually obliged to perform their duties in the contractible activities, but they are free to choose their investments in noncontractible activities and to withhold their services in these activities from the firm. This combination of noncontractible investments and relationship-specificity leads to an ex post multilateral bargaining problem. As in Hart and Moore (1990), we use the Shapley value to determine the division of ex post surplus between the firm and its suppliers. We derive an explicit solution for this division of surplus, which enables us to develop a simple characterization of the equilibrium.

A supplier's expected payoff in the bargaining game determines her willingness to invest in the noncontractible activities. Since she is not the full residual claimant of the output gains derived from

\footnotetext{
${ }^{1}$ Among others, see Klenow and Rodriguez (1997), Hall and Jones (1999) or Caselli (2004) for countries, and Klette (1996), Griliches (1998), Sutton (1998) or Klette and Kortum (2004) for firms.

${ }^{2}$ See, among others, Ethier (1982), Romer (1990) and Grossman and Helpman (1991) for previous uses of this representation. See also the textbook treatment in Barro and Sala-i-Martin (2003). There is a natural relationship between this view of technology and the division of labor within a firm. We investigated this link in the earlier version of the current paper, Acemoglu, Antràs and Helpman (2005), and do not elaborate on it here.

${ }^{3}$ Maskin and Tirole (1999) question whether the presence of nonverifiable actions and unforeseen contingencies necessarily lead to incomplete contracts. Their argument is not central to our analysis because it assumes the presence of "strong" contracting institutions (which, for example, allow contracts to specify sophisticated mechanisms), while in our model a fraction of activities may be noncontractible not because of "technological" reasons but because of weak contracting institutions.
} 
her investments, she tends to underinvest. Greater contractual incompleteness thus reduces supplier investments, making more advanced technologies less profitable. Furthermore, a greater degree of technological complementarity reduces the incentive to choose more advanced technologies; though greater technological complementarity increases equilibrium ex post payoffs to every supplier, it also makes their payoffs less sensitive to their noncontractible investments, discouraging investments and, via this channel, depressing the profitability of more advanced technologies.

An advantage of our framework is its relative tractability. The equilibrium of our model can be represented by a reduced-form profit function for firms given by

$$
A Z F(N)-C(N)-w_{0} N
$$

where $N$ represents the technology level, $C(N)$ is the cost of technology $N, A$ is a measure of aggregate demand or the scale of the market, and $w_{0} N$ corresponds to the value of the $N$ suppliers' outside options. $F(N)$ is an increasing function that captures the positive effect of choosing more advanced technologies on revenue. The effects of contractual incompleteness and technological complementarity are summarized by the variable $Z$. This variable affects productivity and is decreasing in the degree of contract incompleteness and technological complementarity. Moreover, the elasticity of $Z$ with respect to the quality of contracting institutions is higher when there is greater complementarity between intermediate inputs. This last result has important implications for equilibrium industry structure and the patterns of comparative advantage, because it implies that sectors (firms) with greater complementarities between inputs are more "contract dependent".

We use this framework to show that the combination of contractual imperfections and technology choice (or adoption) may have important implications for cross-country income differences, equilibrium organizational forms, and patterns of international specialization and trade.

First, we show that our baseline model can generate sizable differences in productivity from differences in the quality of contracting institutions. In particular, using a range of reasonable values for the key parameters of the model, we find that when the degree of technological complementarity is sufficiently high, relatively modest changes in the fraction of activities that are contractible can lead to large changes in productivity.

Second, we derive a range of implications about the equilibrium organizational form. Our results here show that the combination of weak contracting institutions and credit market imperfections may encourage greater vertical integration.

Third, we present a number of general equilibrium applications of this framework. The general equilibrium interactions result from the fact that an improvement in contracting institutions does not increase the choice of technology in all sectors (firms). Instead, more advanced technologies are chosen in the more contract-dependent sectors. We show that in the context of an open economy, this feature leads to an endogenous structure of comparative advantage. In particular, among countries with identical technological opportunities (production possibility sets), those with better contracting institutions specialize in sectors with greater complementarities among inputs. These predictions are consistent with the recent empirical results presented in Nunn (2005) and Levchenko 
(2003).

As mentioned above, our work is related to two strands of the literature. The first investigates the determinants of firm-level technology (including the division of labor) and includes, among others, Becker and Murphy (1992) and Yang and Borland (1991) on the impact of the extent of the market on the division of labor and Romer (1990) and Grossman and Helpman (1991) on endogenous technological change. None of these studies investigate the effects of contracting institutions on technology choice. The second literature deals with the internal organization of the firm. It includes the papers by Grossman-Hart-Moore discussed above, as well as Klein, Crawford and Alchian (1978) and Williamson (1975, 1985), who emphasize incomplete contracts and hold-up problems. Here, the papers by Stole and Zwiebel (1996a,b) and Bakos and Brynjolfsson (1993) are most closely related to ours. Stole and Zwiebel consider a relationship between a firm and a number of workers whose wages are determined by ex post bargaining according to the Shapley value. They show how the firm may overemploy in order to reduce the bargaining power of the workers and discuss the implications of this framework for a number of organizational design issues. Stole and Zwiebel's framework does not have relationship-specific investments, however, which is at the core of our approach, and they do not discuss the effects of the degree of contractual incompleteness and the degree of complementarity between inputs on the equilibrium technology choice. ${ }^{4}$

Finally, our paper is also related to the literature on the macroeconomic implications of contractual imperfections. A number of papers, most notably Quintin (2001), Amaral and Quintin (2005), Erosa and Hidalgo (2005) and Castro, Clementi and MacDonald (2004), investigate the quantitative impact of contractual and capital market imperfections on aggregate productivity. ${ }^{5}$ Our approach differs from these papers since we focus on technology choice and relationship-specific investments, and because we develop a tractable framework that can be applied in a range of problems. Although we do not undertake a detailed calibration exercise as in these papers, the results in subsection 5.1 suggest that the economic mechanism developed in this paper can lead to quantitatively large effects. Finally, Levchenko (2003), Costinot (2004), Nunn (2005) and Antràs (2005) also generate endogenous comparative advantage across countries from differences in contractual environments. Among these papers, Costinot (2004) is most closely related, since he also develops a model of endogenous comparative advantage based on specialization, though his approach is more reduced-form than our model.

The rest of the paper is organized as follows. Section 2 introduces the basic environment. Section 3 characterizes the equilibrium with complete contracts. Section 4 introduces incomplete contracts into the framework of Section 2, characterizes the equilibrium, and derives the major comparative static results. Section 5 considers a number of applications of our basic framework. Section 6 concludes. Proofs of the main results are provided in the Appendix.

\footnotetext{
${ }^{4}$ Another related paper is Blanchard and Kremer (1997), which studies the impact of inefficient sequential contracting between a firm and its suppliers in a model where output is a Leontief aggregate of the inputs of the suppliers.

${ }^{5}$ Acemoglu and Zilibotti (1999), Martimort and Verdier (2000, 2004) and Francois and Roberts (2003) study the qualitative impact of changes in the internal organization of firms on economic growth.
} 


\section{Technology and Payoffs}

Consider a profit-maximizing firm facing a demand function $q=A p^{-1 /(1-\beta)}$ for its final product, where $q$ denotes quantity and $p$ denotes price. The parameter $\beta \in(0,1)$ determines the elasticity of demand while $A>0$ determines the level of demand or the "market size". The firm treats the demand level $A$ as exogenous. This form of demand can be derived from a constant elasticity of substitution preference structure for differentiated products (see Section 5.3) and it generates a revenue function

$$
R=A^{1-\beta} q^{\beta} .
$$

Production depends on the technology choice of the firm. More advanced (more productive) technologies involve a greater range of intermediate goods and thus a higher degree of specialization. The technology level of the firm is denoted by $N \in \mathbb{R}_{+}$and for each $j \in[0, N], X(j)$ is the quantity of intermediate input $j$. Given technology $N$, the production function of the firm is

$$
q=N^{\kappa+1-1 / \alpha}\left[\int_{0}^{N} X(j)^{\alpha} d j\right]^{1 / \alpha}, 0<\alpha<1, \kappa>0 .
$$

A number of features of this production function are worth noting. First, $\alpha$ determines the degree of complementarity between inputs; since $\alpha \in(0,1)$, the elasticity of substitution between them, $1 /(1-\alpha)$, is always greater than one. Second, we follow Benassy (1998) in introducing the term $N^{\kappa+1-1 / \alpha}$ in front of the integral, which allows us to separately control the elasticity of substitution between inputs and the elasticity of output with respect to the level of the technology. To see this, consider the case where $X(j)=X$ for all $j$. Then the output of technology $N$ is $q=N^{\kappa+1} X$. Consequently, both output and productivity (defined as either $q /(N X)$ or $q / N)$ are independent of $\alpha$ and depend positively on $N$, with elasticity determined by the parameter $\kappa{ }^{6}$

There is a large number of profit-maximizing suppliers that can produce the necessary intermediate goods, each with the same outside option $w_{0}$. For now, $w_{0}$ is taken as given, but it will be endogenized in Section 5.3. We assume that each intermediate input needs to be produced by a different supplier with whom the firm needs to contract. ${ }^{7}$

A supplier assigned to the production of an intermediate input needs to undertake relationshipspecific investments in a unit measure of (symmetric) activities. There is a constant marginal cost of investment $c_{x}$ for each activity. ${ }^{8}$ The production function of intermediate inputs is Cobb-Douglas and symmetric in the activities:

$$
X(j)=\exp \left[\int_{0}^{1} \ln x(i, j) d i\right]
$$

\footnotetext{
${ }^{6}$ In contrast, with the standard specification of the CES production function, without the term $N^{\kappa+1-1 / \alpha}$ in front (i.e., $\kappa=1 / \alpha-1$ ), total output is $q=N^{1 / \alpha} X$, and the two elasticities are governed by the same parameter, $\alpha$.

${ }^{7}$ A previous version of the paper, Acemoglu, Antràs and Helpman (2005), also endogenized the allocation of inputs to suppliers using an augmented model with additional diseconomies of scope.

${ }^{8}$ One can think of $c_{x}$ as the marginal cost of effort; see the formulation of this effort in utility terms in Section 5.3.
} 
where $x(i, j)$ is the level of investment in activity $i$ performed by the supplier of input $j$. This formulation will allow a tractable parameterization of contractual incompleteness in Section 4, whereby a subset of the investments necessary for production will be nonverifiable and thus noncontractible.

Finally, we assume that adopting (and using) a technology $N$ involves $\operatorname{costs} C(N)$, and impose

\section{Assumption 1}

(i) For all $N>0, C(N)$ is twice continuously differentiable, with $C^{\prime}(N)>0$ and $C^{\prime \prime}(N) \geq 0$.

(ii) For all $N>0, N C^{\prime \prime}(N) /\left[C^{\prime}(N)+w_{0}\right]>[\beta(\kappa+1)-1] /(1-\beta)$.

The first part of this assumption is standard; costs are increasing and convex. The second part will ensure a finite and positive choice of $N$.

Let the payment to supplier $j$ consist of two parts: an ex ante payment $\tau(j) \in \mathbb{R}$ before the investment levels $x(i, j)$ take place, and a payment $s(j)$ after the investments. Then, the payoff to supplier $j$, also taking account of her outside option, is

$$
\pi_{x}(j)=\max \left\{\tau(j)+s(j)-\int_{0}^{1} c_{x} x(i, j) d i, w_{0}\right\}
$$

Similarly, the payoff to the firm is

$$
\pi=R-\int_{0}^{N}[\tau(j)+s(j)] d j-C(N),
$$

where $R$ is revenue and the other two terms on the right-hand side represent costs. Substituting (3) and (4) into (2), revenue can be expressed as

$$
R=A^{1-\beta} N^{\beta(\kappa+1-1 / \alpha)}\left[\int_{0}^{N}\left(\exp \left(\int_{0}^{1} \ln x(i, j) d i\right)\right)^{\alpha} d j\right]^{\beta / \alpha} .
$$

\section{Equilibrium under Complete Contracts}

As a benchmark, consider the case of complete contracts (the "first best" from the viewpoint of the firm). With complete contracts, the firm has full control over all investments and pays each supplier her outside option. In analogy to our treatment below of technology adoption under incomplete contracts, consider a game form where the firm chooses a technology level $N$ and makes a contract offer $\left[\{x(i, j)\}_{i \in[0,1]},\{s(j), \tau(j)\}\right]$ for every input $j \in[0, N]$. If a supplier accepts this contract for input $j$, she is obliged to supply $\{x(i, j)\}_{i \in[0,1]}$ as stipulated in the contract in exchange for the payments $\{s(j), \tau(j)\}$. A subgame perfect equilibrium of this game is a strategy combination for the firm and the suppliers such that suppliers maximize (5) and the firm maximizes (6). An equilibrium can be alternatively represented as a solution to the following maximization problem:

$$
\max _{N,\{x(i, j)\}_{i, j},\{s(j), \tau(j)\}_{j}} R-\int_{0}^{N}[\tau(j)+s(j)] d j-C(N)
$$


subject to (7) and the suppliers' participation constraint,

$$
s(j)+\tau(j)-c_{x} \int_{0}^{1} x(i, j) d i \geq w_{0} \text { for all } j \in[0, N] .
$$

Since the firm has no reason to provide rents to the suppliers, it chooses payments $s(j)$ and $\tau(j)$ that satisfy (9) with equality. ${ }^{9}$ Moreover, since the firm's objective function, (8), is (jointly) concave in the investment levels $x(i, j)$ and these investments are all equally costly, the firm chooses the same investment level $x$ for all activities in all intermediate inputs. Now, substituting for (9) in (8), we obtain the following simpler unconstrained maximization problem for the firm:

$$
\max _{N, x} A^{1-\beta} N^{\beta(\kappa+1)} x^{\beta}-c_{x} N x-C(N)-w_{0} N .
$$

$>$ From the first-order conditions of this problem, we obtain:

$$
\begin{gathered}
\left(N^{*}\right)^{\frac{\beta(\kappa+1)-1}{1-\beta}} A \kappa \beta^{1 /(1-\beta)} c_{x}^{-\beta /(1-\beta)}=C^{\prime}\left(N^{*}\right)+w_{0}, \\
x^{*}=\frac{C^{\prime}\left(N^{*}\right)+w_{0}}{\kappa c_{x}} .
\end{gathered}
$$

Equations (11) and (12) can be solved recursively. Given Assumption 1, equation (11) yields a unique solution for $N^{*}$, which, together with (12), yields a unique solution for $x^{*} .{ }^{10}$

When all the investment levels are identical and equal to $x$, output equals $q=N^{\kappa+1} x$. Since $N X=N x$ inputs are used in the production process, we can define productivity as output divided by total input use, $P=N^{\kappa}$. In the case of complete contracts this productivity level is

$$
P^{*}=\left(N^{*}\right)^{\kappa},
$$

which is increasing in the level of technology. In the next section we compare this to equilibrium productivity under incomplete contracts. ${ }^{11}$

The next proposition describes the key properties of the equilibrium (proof in the Appendix).

Proposition 1 Suppose that Assumption 1 holds. Then with complete contracts there exists a unique equilibrium with technology and investment levels $N^{*}>0$ and $x^{*}>0$ given by (11) and (12). Furthermore, this equilibrium satisfies:

$$
\frac{\partial N^{*}}{\partial A}>0, \frac{\partial x^{*}}{\partial A} \geq 0, \frac{\partial N^{*}}{\partial \alpha}=\frac{\partial x^{*}}{\partial \alpha}=0 .
$$

\footnotetext{
${ }^{9}$ With complete contracts, $\tau(j)$ and $s(j)$ are perfect substitutes, so that only the sum $s(j)+\tau(j)$ matters. This will not be the case when contracts are incomplete.

${ }^{10}$ We show in the Appendix that the second-order conditions are satisfied under Assumption 1.

${ }^{11}$ This measure of productivity implicitly assumes that all the investments $x(i, j)$ are measured accurately. There may be some tension between this assumption and the assumption that the cost of these investments is not pecuniary. For this reason in the previous version, we also considered another definition of productivity: output divided by the number of suppliers, $P=q / N=(N)^{\kappa} x$. The ranking of productivity levels between complete and incomplete contracts is the same under both definitions and the quantitative effects of changes in $\mu$ on productivity are smaller with our main definition.
} 
In the case of complete contracts, the size of the market (as parameterized by the demand level $A$ ) has a positive effect on investments by suppliers of intermediate inputs and productivity. The other noteworthy implication of this proposition is that under complete contracts, the level of technology and thus productivity do not depend on the elasticity of substitution between intermediate inputs, $1 /(1-\alpha)$.

\section{Equilibrium under Incomplete Contracts}

\subsection{Incomplete Contracts}

We now consider the same environment under incomplete contracts. We model the imperfection of the contracting institutions by assuming that there exists a $\mu \in[0,1]$ such that, for every intermediate input $j$, investments in activities $0 \leq i \leq \mu$ are observable and verifiable and therefore contractible, while investments in activities $\mu<i \leq 1$ are not contractible. Consequently, a contract stipulates investment levels $x(i, j)$ for the $\mu$ contractible activities, but does not specify the investment levels in the remaining $1-\mu$ noncontractible activities. Instead, suppliers choose their investments in noncontractible activities in anticipation of the ex post distribution of revenue, and may decide to withhold their services in these activities from the firm. We follow the incomplete contracts literature and assume that the ex post distribution of revenue is governed by multilateral bargaining, and, as in Hart and Moore (1990), we adopt the Shapley value as the solution concept for this multilateral bargaining game (more on this below). ${ }^{12}$

The timing of events is as follows:

- The firm adopts a technology $N$ and offers a contract $\left[\left\{x_{c}(i, j)\right\}_{i=0}^{\mu}, \tau(j)\right]$ for every intermediate input $j \in[0, N]$, where $x_{c}(i, j)$ is an investment level in a contractible activity and $\tau(j)$ is an upfront payment to supplier $j$. The payment $\tau(j)$ can be positive or negative.

- Potential suppliers decide whether to apply for the contracts. Then the firm chooses $N$ suppliers, one for each intermediate input $j$.

- All suppliers $j \in[0, N]$ simultaneously choose investment levels $x(i, j)$ for all $i \in[0,1]$. In the contractible activities $i \in[0, \mu]$ they invest $x(i, j)=x_{c}(i, j)$.

- The suppliers and the firm bargain over the division of revenue, and at this stage, suppliers can withhold their services in noncontractible activities.

- Output is produced and sold, and the revenue $R$ is distributed according to the bargaining agreement.

We will characterize a symmetric subgame perfect equilibrium (SSPE for short) of this game, where bargaining outcomes in all subgames are determined by Shapley values.

\footnotetext{
${ }^{12}$ The incomplete contracts literature also assumes that revenues are noncontractible. As is well known, with bilateral contracting or with multilateral contracting and a budget breaker (e.g., Holmström, 1982), contracting on revenues would improve incentives. In our setting, we do not need this assumption, since each firm has a continuum of suppliers and contracts on total revenues would not provide additional investment incentives to suppliers.
} 


\subsection{Definition of Equilibrium and Preliminaries}

Behavior along the SSPE can be described by a tuple $\left\{\tilde{N}, \tilde{x}_{c}, \tilde{x}_{n}, \tilde{\tau}\right\}$ in which $\tilde{N}$ represents the level of technology, $\tilde{x}_{c}$ the investment in contractible activities, $\tilde{x}_{n}$ the investment in noncontractible activities, and $\tilde{\tau}$ the upfront payment to every supplier. That is, for every $j \in[0, \tilde{N}]$ the upfront payment is $\tau(j)=\tilde{\tau}$, and the investment levels are $x(i, j)=\tilde{x}_{c}$ for $i \in[0, \mu]$ and $x(i, j)=\tilde{x}_{n}$ for $i \in(\mu, 1]$. With a slight abuse of terminology, we will denote the SSPE by $\left\{\tilde{N}, \tilde{x}_{c}, \tilde{x}_{n}\right\}$.

The SSPE can be characterized by backward induction. First, consider the penultimate stage of the game, with $N$ as the level of technology, $x_{c}$ as the level of investment in contractible activities. Suppose also that each supplier other than $j$ has chosen a level of investment in noncontractible activities equal to $x_{n}(-j)$ (these are all the same, because we are constructing a symmetric equilibrium), while the investment level in every noncontractible activity by supplier $j$ is $x_{n}(j) .{ }^{13}$ Given these investments, the suppliers and the firm will engage in multilateral Shapley bargaining. Denote the Shapley value of supplier $j$ under these circumstances by $\bar{s}_{x}\left[N, x_{c}, x_{n}(-j), x_{n}(j)\right]$. We derive an explicit formula for this value in the next subsection. For now, note that optimal investment by supplier $j$ implies that $x_{n}(j)$ is chosen to maximize $\bar{s}_{x}\left[N, x_{c}, x_{n}(-j), x_{n}(j)\right]$ minus the cost of investment in noncontractible activities, $(1-\mu) c_{x} x_{n}(j)$. In a symmetric equilibrium, we need $x_{n}(j)=x_{n}(-j)$, or in other words, $x_{n}$ needs to be a fixed-point given by: ${ }^{14}$

$$
x_{n}=\arg \max _{x_{n}(j)} \bar{s}_{x}\left[N, x_{c}, x_{n}, x_{n}(j)\right]-(1-\mu) c_{x} x_{n}(j) .
$$

Equation (14) can be thought of as an "incentive compatibility constraint," with the additional symmetry requirement.

In a symmetric equilibrium with technology $N$, with investment in contractible activities given by $x_{c}$ and with investment in noncontractible activities equal to $x_{n}$, the revenue of the firm is given by $R=A^{1-\beta}\left(N^{\kappa+1} x_{c}^{\mu} x_{n}^{1-\mu}\right)^{\beta}$. Moreover, let $s_{x}\left(N, x_{c}, x_{n}\right)=\bar{s}_{x}\left(N, x_{c}, x_{n}, x_{n}\right)$, then the Shapley value of the firm is obtained as a residual:

$$
s_{q}\left(N, x_{c}, x_{n}\right)=A^{1-\beta}\left(N^{\kappa+1} x_{c}^{\mu} x_{n}^{1-\mu}\right)^{\beta}-N s_{x}\left(N, x_{c}, x_{n}\right) .
$$

Now consider the stage in which the firm chooses $N$ suppliers from a pool of applicants. If suppliers expect to receive less than their outside option, $w_{0}$, this pool is empty. Therefore, for production to take place, the final-good producer has to offer a contract that satisfies the participation constraint of suppliers under incomplete contracts, i.e.,

$$
\bar{s}_{x}\left(N, x_{c}, x_{n}, x_{n}\right)+\tau \geq \mu c_{x} x_{c}+(1-\mu) c_{x} x_{n}+w_{0} \text { for } x_{n} \text { that satisfies (14). }
$$

\footnotetext{
${ }^{13}$ More generally, we would need to consider a distribution of investment levels, $\left\{x_{n}(i, j)\right\}_{i \in(\mu, 1]}$ for supplier $j$, where some of the activities may receive more investment than others. It is straightforward to show, however, that the best deviation for a supplier is to choose the same level of investment in all noncontractible activities. For this reason we save on notation and restrict attention to only such deviations.

${ }^{14}$ This equation should be written with " $\in$ " instead of "=". However, we show below that the fixed point $x_{n}$ in (14) is unique, justifying our use of "=".
} 
In other words, given $N$ and $\left(x_{c}, \tau\right)$, each supplier $j \in[0, N]$ should expect her Shapley value plus the upfront payment to cover the cost of investment in contractible and noncontractible activities and the value of her outside option.

The maximization problem of the firm can then be written as:

$$
\max _{N, x_{c}, x_{n}, \tau} s_{q}\left(N, x_{c}, x_{n}\right)-N \tau-C(N) \text { subject to (14) and (15). }
$$

With no restrictions on $\tau$, the participation constraint (15) will be satisfied with equality; otherwise the firm could reduce $\tau$ without violating (15) and increase its profits. We can therefore solve $\tau$ from this constraint, substitute the solution into the firm's objective function and obtain the simpler maximization problem: ${ }^{15}$

$$
\max _{N, x_{c}, x_{n}} s_{q}\left(N, x_{c}, x_{n}\right)+N\left[\bar{s}_{x}\left(N, x_{c}, x_{n}, x_{n}\right)-\mu c_{x} x_{c}-(1-\mu) c_{x} x_{n}\right]-C(N)-w_{0} N \text { subject to (14). }
$$

The SSPE $\left\{\tilde{N}, \tilde{x}_{c}, \tilde{x}_{n}\right\}$ solves this problem, and the corresponding upfront payment satisfies

$$
\tilde{\tau}=\mu c_{x} \tilde{x}_{c}+(1-\mu) c_{x} \tilde{x}_{n}+w_{0}-\bar{s}_{x}\left(\tilde{N}, \tilde{x}_{c}, \tilde{x}_{n}, \tilde{x}_{n}\right) .
$$

\subsection{Bargaining}

We now derive the Shapley values in this game (see Shapley, 1953, or Osborne and Rubinstein, 1994). In a bargaining game with a finite number of players, each player's Shapley value is the average of her contributions to all coalitions that consist of players ordered below her in all feasible permutations. More explicitly, in a game with $M+1$ players, let $g=\{g(0), g(1), \ldots, g(M)\}$ be a permutation of $0,1,2, \ldots, M$, where player 0 is the firm and players $1,2, \ldots, M$ are the suppliers, and let $z_{g}^{j}=\left\{j^{\prime} \mid g(j)>g\left(j^{\prime}\right)\right\}$ be the set of players ordered below $j$ in the permutation $g$. We denote by $G$ the set of feasible permutations and by $v: G \rightarrow \mathbb{R}$ the value of the coalition consisting of any subset of the $M+1$ players. ${ }^{16}$ Then the Shapley value of player $j$ is

$$
s_{j}=\frac{1}{(M+1) !} \sum_{g \in G}\left[v\left(z_{g}^{j} \cup j\right)-v\left(z_{g}^{j}\right)\right] \text {. }
$$

In the Appendix, we derive the asymptotic Shapley value of Aumann and Shapley (1974), by considering the limit of this expression as the number of players goes to infinity. ${ }^{17}$ Leaving the formal derivation to the Appendix, here we provide a heuristic derivation of this Shapley value.

Suppose the firm has adopted technology $N$, all suppliers provide an amount $x_{c}$ of every con-

\footnotetext{
${ }^{15}$ Note that, as in the case with complete contracts, the firm chooses its technology and investment levels to maximize sale revenues net of total costs. The key difference is that with incomplete contracts, this maximization problem is constrained by the "incentive compatibility" condition (14).

${ }^{16}$ In our game, the value of a coalition equals the amount of revenue this coalition can generate.

${ }^{17}$ More formally, we divide the interval $[0, N]$ into $M$ equally spaced subintervals with all the intermediate inputs in each subinterval of length $N / M$ performed by a single supplier. We then solve for the Shapley value and take the limit of this solution as $M \rightarrow \infty$. See Aumann and Shapley (1974) or Stole and Zwiebel (1996b).
} 
tractible activity, and all suppliers other than $j$ invest $x_{n}(-j)$ in every noncontractible activity, while supplier $j$ invests $x_{n}(j)$. To compute the Shapley value for supplier $j$, first note that the firm is an essential player in this bargaining game (if a coalition does not include the firm, then its output equals zero regardless of its size). Consequently, the supplier $j$ 's marginal contribution is equal to zero when a coalition does not include the firm. When it does include the firm and a measure $n$ of suppliers, the marginal contribution of supplier $j$ is $m(j, n)=\partial \bar{R} / \partial n$, where

$$
\bar{R}=A^{1-\beta} N^{\beta(\kappa+1-1 / \alpha)}\left[\int_{0}^{n}\left(\exp \left(\int_{0}^{1} \ln x(i, k) d i\right)\right)^{\alpha} d k\right]^{\beta / \alpha}
$$

is the revenue derived from the employment of $n$ inputs with technology $N$, and the last input $k=n$ is provided by supplier $j$. Since $x(i, k)=x_{c}$ for all $0 \leq i \leq \mu$ and all $0 \leq k \leq n, x(i, k)=x_{n}(-j)$ for all $\mu<i \leq 1$ and all $0 \leq k<n$, and $x(i, k)=x_{n}(j)$ for all $\mu<i \leq 1$ and $k=n$, evaluating the previous expression enables us to write the marginal contribution of supplier $j$ as

$$
m(j, n)=\frac{\beta}{\alpha} A^{1-\beta} N^{\beta(\kappa+1-1 / \alpha)}\left[\frac{x_{n}(j)}{x_{n}(-j)}\right]^{(1-\mu) \alpha} x_{c}^{\beta \mu} x_{n}(-j)^{\beta(1-\mu)} n^{(\beta-\alpha) / \alpha} .
$$

The Shapley value of supplier $j$ is the average of her marginal contributions to coalitions that consist of players ordered below her in all feasible orderings. A supplier that has a measure $n$ of players ordered below her has a marginal contribution of $m(j, n)$ if the firm is ordered below her (probability $n / N)$ and 0 otherwise (probability $1-n / N$ ). Averaging over all possible orderings of the players and using (18), we obtain:

$$
\begin{aligned}
\bar{s}_{x}\left[N, x_{c}, x_{n}(-j), x_{n}(j)\right] & =\frac{1}{N} \int_{0}^{N}\left(\frac{n}{N}\right) m(j, n) d n \\
& =(1-\gamma) A^{1-\beta}\left[\frac{x_{n}(j)}{x_{n}(-j)}\right]^{(1-\mu) \alpha} x_{c}^{\beta \mu} x_{n}(-j)^{\beta(1-\mu)} N^{\beta(\kappa+1)-1},
\end{aligned}
$$

where

$$
\gamma \equiv \frac{\alpha}{\alpha+\beta}
$$

We therefore obtain the following lemma (see the Appendix for the formal proof):

Lemma 1 Suppose that supplier $j$ invests $x_{n}(j)$ in her noncontractible activities, all the other suppliers invest $x_{n}(-j)$ in their noncontractible activities, every supplier invests $x_{c}$ in her contractible activities, and the level of technology is $N$. Then the Shapley value of supplier $j$ is

$$
\bar{s}_{x}\left[N, x_{c}, x_{n}(-j), x_{n}(j)\right]=(1-\gamma) A^{1-\beta}\left[\frac{x_{n}(j)}{x_{n}(-j)}\right]^{(1-\mu) \alpha} x_{c}^{\beta \mu} x_{n}(-j)^{\beta(1-\mu)} N^{\beta(\kappa+1)-1},
$$

where $\gamma$ is defined in (19).

A number of features of (20) are worth noting. First, in equilibrium, all suppliers invest equally 
in all the noncontractible activities, i.e., $x_{n}(j)=x_{n}(-j)=x_{n}$, and so

$$
s_{x}\left(N, x_{c}, x_{n}\right)=\bar{s}_{x}\left(N, x_{c}, x_{n}, x_{n}\right)=(1-\gamma) A^{1-\beta} x_{c}^{\beta \mu} x_{n}^{\beta(1-\mu)} N^{\beta(\kappa+1)-1}=(1-\gamma) \frac{R}{N},
$$

where $R=A^{1-\beta} x_{c}^{\beta \mu} x_{n}^{\beta(1-\mu)} N^{\beta(\kappa+1)}$ is the total revenue of the firm. Thus, the joint Shapley value of the suppliers, $N s_{x}\left(N, x_{c}, x_{n}\right)$, equals the fraction $1-\gamma$ of the revenue, and the firm receives the remaining fraction $\gamma$, i.e.,

$$
s_{q}\left(N, x_{c}, x_{n}\right)=\gamma A^{1-\beta} x_{c}^{\beta \mu} x_{n}^{\beta(1-\mu)} N^{\beta(\kappa+1)}=\gamma R .
$$

This is a relatively simple rule for the division of revenue between the firm and its suppliers.

Second, the derived parameter $\gamma \equiv \alpha /(\alpha+\beta)$ represents the bargaining power of the firm; it is increasing in $\alpha$ and decreasing in $\beta$. A higher elasticity of substitution between intermediate inputs, i.e., a higher $\alpha$, raises the firm's bargaining power, because it makes every supplier less essential in production and therefore raises the share of revenue appropriated by the firm. In contrast, a higher elasticity of demand for the final good, i.e., higher $\beta$, reduces the firm's bargaining power, because, for any coalition, it reduces the marginal contribution of the firm to the coalition's payoff as a fraction of revenue. ${ }^{18}$

Furthermore, when $\alpha$ is smaller, $\bar{s}_{x}\left[N, x_{c}, x_{n}(-j), x_{n}(j)\right]$ is more concave with respect to $x_{n}(j)$, because greater complementary between the intermediate inputs implies that a given change in the relative employment of two inputs has a larger impact on their relative marginal products. The impact of $\alpha$ on the concavity of $\bar{s}_{x}(\cdot)$ will play an important role in the following results. The parameter $\beta$, on the other hand, affects the concavity of revenue in output (see (2)) but has no effect on the concavity of $\bar{s}_{x}$ with respect to $x_{n}(j)$, because with a continuum of suppliers, a single supplier has an infinitesimal effect on output.

\subsection{Equilibrium}

To characterize a SSPE, we first derive the incentive compatibility constraint using (14) and (20):

$$
x_{n}=\arg \max _{x_{n}(j)}(1-\gamma) A^{1-\beta}\left[\frac{x_{n}(j)}{x_{n}}\right]^{(1-\mu) \alpha} x_{c}^{\beta \mu} x_{n}^{\beta(1-\mu)} N^{\beta(\kappa+1)-1}-c_{x}(1-\mu) x_{n}(j) .
$$

\footnotetext{
${ }^{18}$ To clarify the effects of $\alpha$ and $\beta$ on $\gamma$, let us return to the derivation in the text and note that the marginal contribution of the firm to a coalition of $n$ suppliers, each one investing $x_{c}$ in contractible activities and $x_{n}$ in noncontractible activities, can be expressed as

$$
m_{q}(n)=A^{1-\beta} N^{\beta(\kappa+1)} x_{c}^{\beta \mu} x_{n}^{\beta(1-\mu)}\left(\frac{n}{N}\right)^{\beta / \alpha}=\left(\frac{n}{N}\right)^{\beta / \alpha} R
$$

where $R=A^{1-\beta} N^{\beta(\kappa+1)} x_{c}^{\beta \mu} x_{n}^{\beta(1-\mu)}$ is the equilibrium level of the revenue (when $\left.n=N\right)$. The expression $(n / N)^{\beta / \alpha}$ is decreasing in $\beta$ and increasing in $\alpha$ for all $n<N$. The parameter $\gamma$ is given by the average of the $(n / N)^{\beta / \alpha}$ terms,

$$
\gamma=\frac{1}{N} \int_{0}^{N}\left(\frac{n}{N}\right)^{\beta / \alpha} d n=\frac{\alpha}{\alpha+\beta},
$$
}

and is also decreasing in $\beta$ and increasing in $\alpha$. 
Relative to the producer's first-best choice characterized above, we see two differences. First, the term $(1-\gamma)$ implies that the supplier is not the full residual claimant of the return from her investment in noncontractible activities and thus underinvests in these activities. Second, as discussed above, multilateral bargaining distorts the perceived concavity of the private return relative to the social return. Using the first-order condition of this problem and solving for the fixed point by substituting $x_{n}(j)=x_{n}$ yields a unique $x_{n}$ :

$$
x_{n}=\bar{x}_{n}\left(N, x_{c}\right) \equiv\left[\alpha(1-\gamma)\left(c_{x}\right)^{-1} x_{c}^{\beta \mu} A^{1-\beta} N^{\beta(\kappa+1)-1}\right]^{1 /[1-\beta(1-\mu)]} .
$$

Note that $\bar{x}_{n}\left(N, x_{c}\right)$ is increasing in $x_{c}$; since the marginal productivity of an activity rises with investment in other activities, investments in contractible and noncontractible activities are complements. ${ }^{19}$ Another implication of (23) is that investment in noncontractible activities is increasing in $\alpha$. Mathematically, this follows from the fact that $\alpha(1-\gamma)=\alpha \beta /(\alpha+\beta)$ is increasing in $\alpha$. However, the economics of this relationship is the outcome of two opposing forces. The share of the suppliers in revenue, $(1-\gamma)$, is decreasing in $\alpha$, because greater substitution between the intermediate inputs reduces the suppliers' ex post bargaining power. But a greater level of $\alpha$ also reduces the concavity of $\bar{s}_{x}(\cdot)$ in $x_{n}$, increasing the marginal reward from investing further in noncontractible activities. Because the latter effect dominates, $x_{n}$ is increasing in $\alpha$.

Now, using (21), (22) and (23), the firm's optimization problem (16) can be expressed as

$$
\max _{N, x_{c}} A^{1-\beta}\left[x_{c}^{\mu} \bar{x}_{n}\left(N, x_{c}\right)^{1-\mu}\right]^{\beta} N^{\beta(\kappa+1)}-c_{x} N \mu x_{c}-c_{x} N(1-\mu) \bar{x}_{n}\left(N, x_{c}\right)-C(N)-w_{0} N,
$$

where $\bar{x}_{n}\left(N, x_{c}\right)$ is defined in (23). Substituting (23) into (24) and differentiating with respect to $N$ and $x_{c}$ results in two first-order conditions, which yield a unique solution $\left(\tilde{N}, \tilde{x}_{c}\right)$ to $(24):^{20}$

$$
\begin{aligned}
& \tilde{N}^{\frac{\beta(\kappa+1)-1}{1-\beta}} A \kappa \beta^{\frac{1}{1-\beta}} c_{x}^{-\frac{\beta}{1-\beta}}\left[\frac{1-\alpha(1-\gamma)(1-\mu)}{1-\beta(1-\mu)}\right]^{\frac{1-\beta(1-\mu)}{1-\beta}}\left[\beta^{-1} \alpha(1-\gamma)\right]^{\frac{\beta(1-\mu)}{1-\beta}}=C^{\prime}(\tilde{N})+w_{0} \\
& \tilde{x}_{c}=\frac{C^{\prime}(\tilde{N})+w_{0}}{\kappa c_{x}} .
\end{aligned}
$$

As in the complete contracts case, these two conditions determine the equilibrium recursively. First, (25) gives $\tilde{N}$, and then given $\tilde{N},(26)$ yields $\tilde{x}_{c}$. Moreover, using (23), (25), and (26) gives

\footnotetext{
${ }^{19}$ The effect of $N$ on $x_{n}$ is ambiguous, since investment in noncontractible activities declines with the level of technology when $\beta(\kappa+1)<1$ and increases with $N$ when $\beta(\kappa+1)>1$. This is because an increase in $N$ has two opposite effects on a supplier's incentives to invest; a greater number of inputs increases the marginal product of investment due to the "love for variety" embodied in the technology, but at the same time, the bargaining share of a supplier, $(1-\gamma) / N$, declines with $N$. For large values of $\kappa$ the former effect dominates, while for small values of $\kappa$ the latter dominates.

${ }^{20}$ See the Appendix for a more detailed derivation and for the second-order conditions.
} 
the level of investment in noncontractible activities as

$$
\tilde{x}_{n}=\frac{\alpha(1-\gamma)[1-\beta(1-\mu)]}{\beta[1-\alpha(1-\gamma)(1-\mu)]}\left(\frac{C^{\prime}(\tilde{N})+w_{0}}{\kappa c_{x}}\right) .
$$

Comparing (12) to (26), we see that for a given $N$ the implied level of investment in contractible activities under incomplete contracts, $\tilde{x}_{c}$, is identical to the investment level in contractible activities under complete contracts, $x^{*}$. This highlights the fact that differences in the investment in contractible activities between these economic environments only result from differences in technology adoption. In fact, comparing (11) with (25), we see that $\tilde{N}$ and $N^{*}$ differ only because of the two bracketed terms on the left-hand side of (25). These represent the distortions created by bargaining between the firm and its suppliers. Intuitively, technology adoption is distorted because incomplete contracts reduce investment in noncontractible activities below the level of investment in contractible activities and this "underinvestment" reduces the profitability of technologies with high $N$. As $\mu \rightarrow 1$ (and contractual imperfections disappear), both of these bracketed terms on the left-hand side of $(25)$ go to 1 and $\left(\tilde{N}, \tilde{x}_{c}\right) \rightarrow\left(N^{*}, x^{*}\right) .{ }^{21}$

The impact of incomplete contracts on productivity follows directly from their effect on the choice of technology. In particular, productivity under incomplete contracts, $\tilde{P}=\tilde{N}^{\kappa}$, is always lower than $P^{*}$ as given in $(13)$, since $\tilde{N}<N^{*}$.

\subsection{Implications of Incomplete Contracts}

We now provide a number of comparative static results on the SSPE under incomplete contracts, and compare the incomplete-contracts equilibrium technology and investment levels to the equilibrium under complete contracts. The comparative static results are facilitated by the block-recursive structure of the equilibrium; any change in $A, \mu$ or $\alpha$ that increases the left-hand side of (25) also increase $\tilde{N}$, and the effect on $\tilde{x}_{c}$ and $\tilde{x}_{n}$ can then be obtained from (26) and (27). The main results are provided in the next proposition (proof in the Appendix).

Proposition 2 Suppose that Assumption 1 holds. Then there exists a unique SSPE under incomplete contracts, $\left\{\tilde{N}, \tilde{x}_{c}, \tilde{x}_{n}\right\}$, characterized by (25), (26) and (27). Furthermore, $\left\{\tilde{N}, \tilde{x}_{c}, \tilde{x}_{n}\right\}$ satisfies $\tilde{N}, \tilde{x}_{c}, \tilde{x}_{n}>0$,

$$
\tilde{x}_{n}<\tilde{x}_{c}
$$

\footnotetext{
${ }^{21}$ Note that as $\mu \rightarrow 1$ the investment level $\tilde{x}_{n}$ does not converge to $x^{*}$, because the effect of distortions on the noncontractible activities does not go to zero. What goes to zero, however, is the importance of noncontractible activities in the production of final goods.
} 


$$
\begin{aligned}
& \frac{\partial \tilde{N}}{\partial A}>0, \frac{\partial \tilde{x}_{c}}{\partial A} \geq 0, \frac{\partial \tilde{x}_{n}}{\partial A} \geq 0 \\
& \frac{\partial \tilde{N}}{\partial \mu}>0, \frac{\partial \tilde{x}_{c}}{\partial \mu} \geq 0, \frac{\partial\left(\tilde{x}_{n} / \tilde{x}_{c}\right)}{\partial \mu}>0 \\
& \frac{\partial \tilde{N}}{\partial \alpha}>0, \frac{\partial \tilde{x}_{c}}{\partial \alpha} \geq 0, \frac{\partial\left(\tilde{x}_{n} / \tilde{x}_{c}\right)}{\partial \alpha}>0 .
\end{aligned}
$$

The main results in this proposition are intuitive. Suppliers invest less in noncontractible activities than in contractible activities, in particular,

$$
\frac{\tilde{x}_{n}}{\tilde{x}_{c}}=\frac{\alpha(1-\gamma)[1-\beta(1-\mu)]}{\beta[1-\alpha(1-\gamma)(1-\mu)]}<1,
$$

which follows from equations (26) and (27) and from the fact that $\alpha(1-\gamma)=\alpha \beta /(\alpha+\beta)<\beta$ (recall (19)). Intuitively, the firm is the full residual claimant of the return to investments in contractible activities and it dictates these investments in the contract. In contrast, investments in noncontractible activities are decided by the suppliers, who are not the full residual claimants of the returns generated by these investments (recall (21)) and thus underinvest in these activities.

In addition, the level of technology and investments in both contractible and noncontractible activities are increasing in the size of the market, in the fraction of contractible activities (quality of contracting institutions), and in the elasticity of substitution between intermediate inputs. ${ }^{22}$ The impact of the size of the market is intuitive; a greater $A$ makes production more profitable and thus increases investments and equilibrium technology. Better contracting institutions, on the other hand, imply that a greater fraction of activities receive the higher investment level $\tilde{x}_{c}$ rather than $\tilde{x}_{n}<\tilde{x}_{c}$. This makes the choice of a more advanced technology more profitable. A higher $N$, in turn, increases the profitability of further investments in $\tilde{x}_{c}$ and $\tilde{x}_{n}$. Better contracting institutions also close the (proportional) gap between $\tilde{x}_{c}$ and $\tilde{x}_{n}$ because with a higher fraction of contractible activities, the marginal return to investment in noncontractible activities is also higher.

A higher $\alpha$, i.e., lower complementarity between intermediate inputs, also increases technology choices and investments. The reason is related to the discussion in the previous subsection where it was shown that a higher $\alpha$ reduces the share of each supplier but also makes $\bar{s}_{x}(\cdot)$ less concave. Because the latter effect dominates, a lower degree of complementarity increases supplier investments and makes the adoption of more advanced technologies more profitable.

The reduced-form profit function depicted in equation (1) in the Introduction can also be derived at this point. Combining (23), (24), and the condition for the firm's optimal choice of $x_{c}$, the firm's payoff can be expressed as (see the Appendix):

$$
\pi=A Z(\alpha, \mu) N^{1+\frac{\beta(\kappa+1)-1}{1-\beta}}-C(N)-w_{0} N
$$

\footnotetext{
${ }^{22}$ Equation (13) above implies that the measure of productivity, $P=q /(N X)$, has the same comparative statics as technology. The same results also apply if we were to define productivity as $P=q / N$, that is, as output divided by the number of suppliers. Yet, in this case firms operating under different contracting institutions would have different productivity levels not only because they choose different levels of technology, but also because they have different investment levels. See Acemoglu, Antràs and Helpman (2005) for more details on this point.
} 
where

$$
Z(\alpha, \mu) \equiv(1-\beta) \beta^{\frac{\beta \mu}{1-\beta}}[\alpha(1-\gamma)]^{\frac{\beta(1-\mu)}{1-\beta}}\left[\frac{1-\alpha(1-\gamma)(1-\mu)}{1-\beta(1-\mu)}\right]^{\frac{1-\beta(1-\mu)}{1-\beta}}\left(c_{x}\right)^{-\frac{\beta}{1-\beta}}
$$

represents a measure of "derived efficiency" and captures the distortions arising from incomplete contracts. The extent of these distortions depends on the model's parameters as shown in Proposition 2. In addition, we have the following lemma (proof in the Appendix):

Lemma 2 Suppose that Assumption 1 holds. Let $\zeta_{\mu}(\alpha, \mu) \equiv(\mu \times \partial Z(\alpha, \mu) / \partial \mu) / Z(\alpha, \mu)$ be the elasticity of $Z(\alpha, \mu)$ with respect to $\mu$ and let let $\zeta_{\alpha}(\alpha, \mu) \equiv(\alpha \times \partial Z(\alpha, \mu) / \partial \alpha) / Z(\alpha, \mu)$ be the elasticity of $Z(\alpha, \mu)$ with respect to $\alpha$. Then, we have that

1. $\zeta_{\mu}(\alpha, \mu)>0$ and $\zeta_{\alpha}(\alpha, \mu)>0$; and

2. $\partial \zeta_{\mu}(\alpha, \mu) / \partial \alpha<0$ and $\partial \zeta_{\alpha}(\alpha, \mu) / \partial \mu<0$.

Part 1 of this lemma implies that better contracting institutions and greater substitutability between intermediate inputs lead to higher levels of $Z(\alpha, \mu)$. Part 2, on the other hand, implies that the proportional increase in $Z(\alpha, \mu)$ in response to an improvement in contracting institutions is greater when there is more technological complementarity between intermediate inputs. The intuition is that contract incompleteness is more damaging to technologies with greater complementarities, because there are more significant investment distortions in this case. This last result implies that sectors with greater complementarities are more "contract dependent" and will play an important role in the general equilibrium analysis in Section 5.3.

Finally, to compare the complete and incomplete contracts equilibria, recall that they both lead to the same allocation as $\mu \rightarrow 1$. Together with Proposition 2, this implies (proof in the Appendix):

Proposition 3 Suppose that Assumption 1 holds. Let $\left\{\tilde{N}, \tilde{x}_{c}, \tilde{x}_{n}\right\}$ be the unique SSPE with incomplete contracts and let $\left\{N^{*}, x^{*}\right\}$ be the unique equilibrium with complete contracts. Then

$$
\tilde{N}<N^{*} \text { and } \tilde{x}_{n}<\tilde{x}_{c} \leq x^{*} \text {. }
$$

This proposition implies that since incomplete contracts lead to the choice of less advanced (lower $N$ ) technologies, ${ }^{23}$ they also reduce productivity and investments in contractible and noncontractible activities.

\footnotetext{
${ }^{23}$ It is useful to contrast this result with the overemployment result in Stole and Zwiebel (1996a,b). There are two important differences between our model and theirs. First, our model features investments in noncontractible activities, which are absent in Stole and Zwiebel. Second, Stole and Zwiebel assume that if a worker is not in the coalition of the bargaining game, she receives no payment whatsoever. Since in our model investment in noncontractible activities is not verifiable, a supplier receives the upfront payment $\tau$ independently of whether she is in the coalition, and she receives only the rest of the payment, i.e., $s_{x}$, through bargaining. The treatment of the outside option in Stole and Zwiebel is essential for their overemployment result (see de Fontenay and Gans, 2003).
} 


\section{Applications}

We next discuss a number of applications of the basic framework developed so far. These applications emphasize both the potential quantitative effects of our main mechanism and its implications for equilibrium organizational choices and endogenous patterns of comparative advantage.

\subsection{Quantitative Implications}

We first investigate whether our baseline model can generate significant productivity differences from cross-country variation in contracting institutions. Our purpose here is not to undertake a full-fledged calibration, but to give a sense of the empirical implications of our baseline model for plausible parameter values. To obtain closed-form solutions for the equilibrium value of $N$ and for the productivity measure $P=N^{\kappa}$, we assume that the function $C(N)$ is linear in $N, C(N)=\phi N$. Equation (25) then implies: ${ }^{24}$

$$
\tilde{P}=\left(\frac{A \kappa}{\phi+w_{0}}\right)^{\frac{\kappa(1-\beta)}{1-\beta(\kappa+1)}} \beta^{\frac{\kappa}{1-\beta(\kappa+1)}} c_{x}^{-\frac{\beta \kappa}{1-\beta(\kappa+1)}}\left[\frac{1-\alpha(1-\gamma)(1-\mu)}{1-\beta(1-\mu)}\right]^{\frac{\kappa(1-\beta(1-\mu))}{1-\beta(\kappa+1)}}\left[\frac{\alpha(1-\gamma)}{\beta}\right]^{\frac{\kappa \beta(1-\mu)}{1-\beta(\kappa+1)}} .
$$

Our focus is on the impact of the quality of contracting institutions, $\mu$, on firm-level productivity. For this purpose, consider the ratio of productivity in two economies with the fraction of contractible tasks given by $\mu_{1}$ and $\mu_{0}<\mu_{1}$,

$$
\frac{\tilde{P}\left(\mu_{1}\right)}{\tilde{P}\left(\mu_{0}\right)}=\frac{\left[\frac{1-\alpha(1-\gamma)\left(1-\mu_{1}\right)}{1-\beta\left(1-\mu_{1}\right)}\right]^{\frac{\kappa\left(1-\beta\left(1-\mu_{1}\right)\right)}{1-\beta(\kappa+1)}}}{\left[\frac{1-\alpha(1-\gamma)\left(1-\mu_{0}\right)}{1-\beta\left(1-\mu_{0}\right)}\right]^{\frac{\kappa\left(1-\beta\left(1-\mu_{0}\right)\right)}{1-\beta(\kappa+1)}}}\left[\beta^{-1} \alpha(1-\gamma)\right]^{\frac{\kappa \beta\left(\mu_{0}-\mu_{1}\right)}{1-\beta(\kappa+1)}}
$$

where recall that $\gamma \equiv \alpha /(\alpha+\beta)$. Equation (31) shows that the proportional impact of $\mu$ on aggregate productivity only depends on the values of the parameters $\alpha, \beta$, and $\kappa .^{25}$

The parameter $\beta$ governs the elasticity of substitution between final-good varieties and also determines the markup charged by final-good producers. In our benchmark simulation, we set this parameter equal to 0.75 . This implies an elasticity of substitution between final-good varieties equal to 4, which corresponds to the mean elasticity estimated by Broda and Weinstein (2006) using U.S. import data. This value of $\beta$ also implies a markup over marginal cost equal to $\beta^{-1}-1 \simeq 0.33$, which is comfortably within the range of available estimates. ${ }^{26}$

\footnotetext{
${ }^{24}$ Recall from the discussion in footnote 11 that there are alternative ways of measuring productivity in the model, and which one of these is more appropriate will depend on how productivity is measured in practice. If, for example, we were to measure productivity as output divided by the number of suppliers, the impact of improvements in contracting institutions on productivity would be even larger, because such improvements raise investment in contractible and noncontractible activities as well.

${ }^{25}$ In deriving equation (31), we assume that the remaining parameters are held fixed when $\mu$ changes. We should thus interpret our results as reflecting the partial-equilibrium response of productivity to changes in contractibility.

${ }^{26}$ On the lower side, Morrison (1992) finds markups ranging from 0.1 in 1961 to 0.29 in 1970, while on the higher side Rotemberg and Woodford (1991) use an estimate of 0.6. See Basu (1995) for a discussion of the sensitivity of the estimated markups to model specification and calibration. It is interesting to note that Morrison (1995) finds markups between 0.1 and 0.39 in Japan and 0.1 and 0.2 in Canada, Roberts (1996) finds average markups between
} 


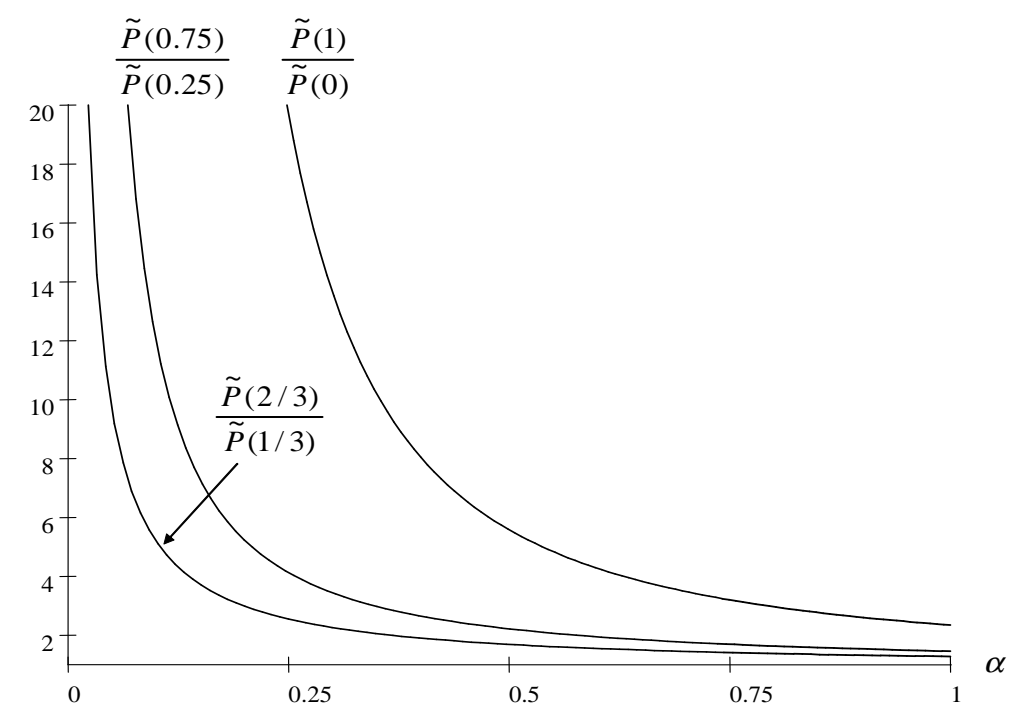

Figure 1: Relative productivity for $\beta=0.75$ and $\kappa=0.25$

We choose the parameter $\kappa$ to match Bils and Klenow's (2001) estimates of variety growth in the U.S. economy from BLS data on expenditures on different types of goods. Their findings indicate that the growth of the number of varieties in the U.S. economy was around $2 \%$ per annum between 1959 and 1999. If we assume that the variety of intermediate goods grew at the same rate over this period and also that this variety growth was the main source of productivity (TFP) growth in the U.S. economy, then we can use our model to back out a value of $\kappa$. We take the rate of TFP growth per annum to be $0.5 \%$ and use the fact that productivity is given by $P=N^{\kappa}$. This implies that $\left(N_{t+1}^{\kappa}-N_{t}^{\kappa}\right) / N_{t}^{\kappa}=0.005$ and generates a value of $\kappa=\ln (1.005) / \ln (1.02) \simeq 0.25 .^{27}$

The value of $\alpha$ is harder to pin down. Since the main theoretical ideas in the paper relate to complementarity between different inputs, a low value of $\alpha$ may be natural. Nevertheless, since we have no direct way of relating $\alpha$ to aggregate data, we show the implications of changes in the quality of contracting institutions for different values of $\alpha$.

Figure 1 depicts the value of the ratio in (31) for different values of $\alpha$ when $\beta=0.75$ and $\kappa=0.25$. We consider three experiments. The middle curve depicts a shift from $\mu_{0}=0.25$ to $\mu_{1}=0.75$, the lowest curve depicts a more modest increase from $\mu_{0}=1 / 3$ to $\mu_{1}=2 / 3$, while the highest curve illustrates the most extreme shift from $\mu_{0}=0$ to $\mu_{1}=1$.

The patterns in Figure 1 confirm the results in Lemma 2 and show that the impact of an increase in $\mu$ on productivity is larger for lower values of $\alpha$. More importantly, the figure shows that the

0.22 and 0.3 in Colombia, and Grether (1996) finds average markups between 0.34 and 0.37 in Mexico.

${ }^{27}$ A somewhat different number is obtained from Broda and Weinstein's (2006) estimate that the number of varieties in U.S. imports between 1972 and 2001 grew at an annual rate of $3.7 \%$. Using the same formula, this would imply a value of 0.135 for $\kappa$. Note, however, that this is likely to be an overestimate of variety growth and thus an underestimate of $\kappa$ because Broda and Weinstein consider the same good imported from different countries as different varieties, and the number of exporting countries to the U.S. increased over time. For this reason, we choose $\kappa=0.25$ as our benchmark parameter value. 


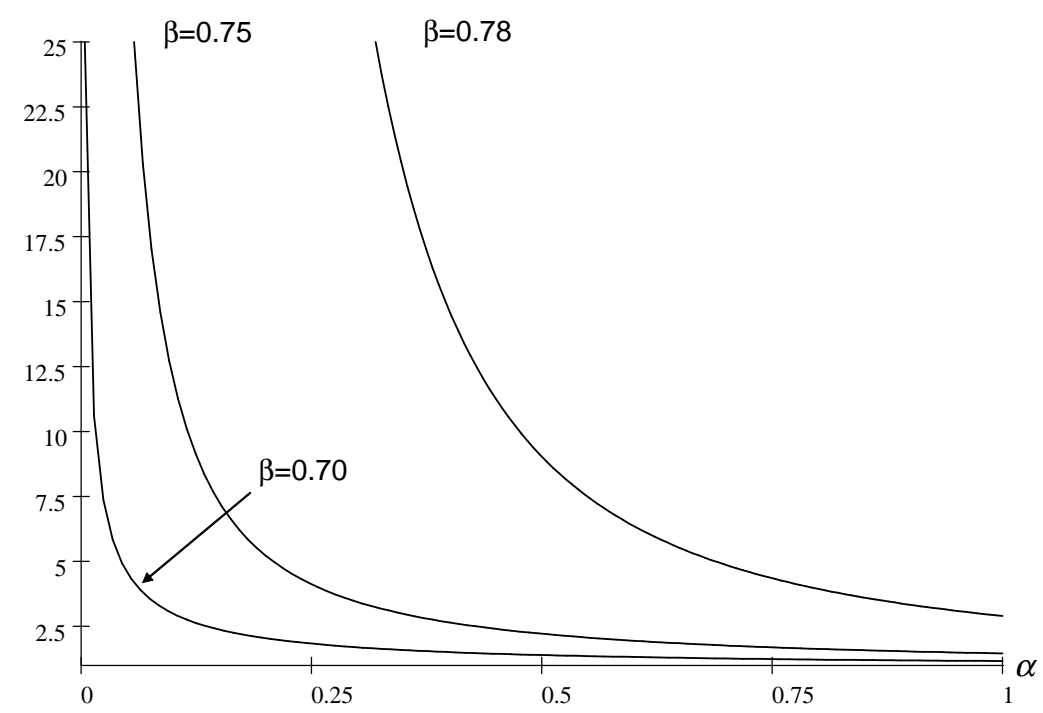

Figure 2: Relative productivity $\tilde{P}(0.75) / \tilde{P}(0.25)$ for $\kappa=0.25$ and alternative $\beta$ 's.

quantitative effects can be sizable for a large range of values of $\alpha$. For example, when $\alpha=0.25$, productivity increases by a factor of 2.5, 4.1 and 19.7 in the three experiments. However, as $\alpha$ increases and intermediate inputs become more substitutable, the magnitude of the quantitative effects diminishes. For example, when $\alpha=0.75$, productivity increases, respectively, by a factor of 1.4, 1.7, and 3.2 in the three cases. Though smaller, these are still sizable effects.

We next discuss the sensitivity of these quantitative results to alternative parameter values. While markup estimates are consistent with a value of $\beta=0.75$, other evidence suggests somewhat higher or lower values of $\beta$. Figure 2 shows the results of an increase in $\mu$ from 0.25 to 0.75 when $\beta=0.78$ and when $\beta=0.7$, while leaving $\kappa$ at $0.25{ }^{28}$ The figure shows that the quantitative effects are considerably larger in the case in which $\beta=0.78$, even for large values of $\alpha$. For example, with $\alpha=0.75$, the improvement in contracting institutions leads to a proportional increase in productivity of 4.4 , which is a very sizable effect. Setting $\beta$ equal to 0.7 reduces the effect of improved contractibility on productivity, but for $\alpha=0.25$ we still find that the experiment increases productivity by a factor of 1.8 .

Finally, Figure 3 shows the results for alternative values of $\kappa, \kappa=0.2$ and $\kappa=0.3$, while holding $\beta$ at 0.75 . When $\kappa=0.2$, the estimated effects are somewhat smaller than in our benchmark simulation, but still sizable; with low values of $\alpha$, the impact of an improvement in the quality of contracting institutions on productivity is still large. For example, with $\alpha=0.25$, productivity increases by a factor of 2.0. On the other hand, greater values of $\kappa$ lead to more significant responses of productivity to increases in $\mu$. Even for a very high value of $\alpha$ such as 0.75 , productivity increases by a factor of 4.8 as $\mu$ increases from 0.25 to 0.75 .

Overall, this simple quantitative evaluation suggests that the mechanism highlighted in our

\footnotetext{
${ }^{28}$ Only values of $\beta$ less than 0.8 are consistent with Assumption 1 combined with $\kappa=0.25$.
} 


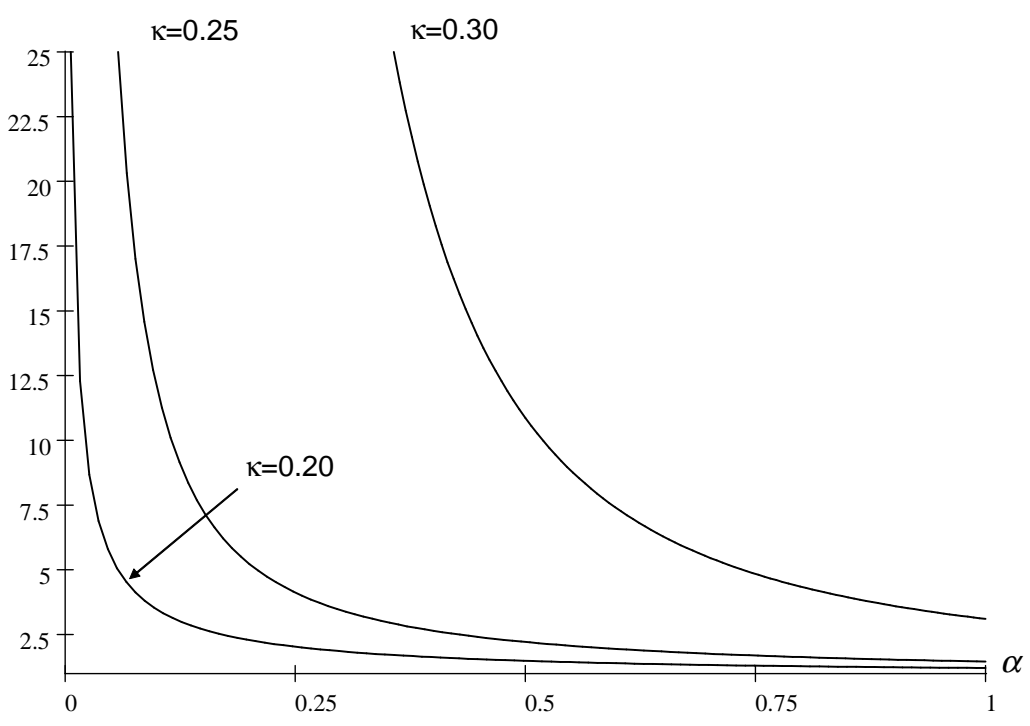

Figure 3: Relative productivity $\tilde{P}(0.75) / \tilde{P}(0.25)$ for $\beta=0.75$ and alternative $\kappa$ 's.

model is capable of generating quantitatively sizable effects from relatively modest variations in contracting institutions.

\subsection{Choice of Organizational Forms}

An important insight of the incomplete contracts literature, and especially of Grossman and Hart (1986) and Hart and Moore (1990), is to consider organizational forms (and the ownership of assets) as a choice variable affecting ex ante investments. Our framework is tractable enough to allow these considerations and can be used to discuss issues of vertical integration versus outsourcing, and how the employment relationship between the firm and its suppliers should be organized. We now briefly discuss how this can be done. A more detailed treatment is presented in our working paper version, Acemoglu, Antràs and Helpman (2005).

We have so far assumed that the threat point of a supplier is not to deliver the noncontractible activities, which - in view of the Cobb-Douglas structure of the production function of the intermediate input in (4) - is equivalent to assuming that the supplier does not deliver $X(j)$ at all. Assume instead that the threat point of a supplier is not to deliver a fraction $1-\delta$ of her $X(j)$, where $0 \leq \delta \leq 1$. The magnitude of $\delta$ depends, among other things, on whether the supplier is an employee of the firm or an outside contractor. Our analysis above corresponds to the case $\delta=0$. The following lemma generalizes the Shapley value to the case where $\delta>0$ (proof in the Appendix):

Lemma 3 Suppose that supplier $j$ invests $x_{n}(j)$ in her noncontractible activities, all the other suppliers invest $x_{n}(-j)$ in their noncontractible activities, every supplier invests $x_{c}$ in her contractible activities, and the level of technology is $N$. Then the Shapley value of supplier $j$ is given 
by (20), where

$$
\gamma \equiv \frac{\alpha\left(1-\delta^{\alpha+\beta}\right)}{(\alpha+\beta)\left(1-\delta^{\alpha}\right)} .
$$

Lemma 3 implies that the formula for the Shapley value is the same as before, except that now the firm's share in the bargaining game, $\gamma$, depends on $\delta$. Clearly, $\gamma$ in (32) equals $\gamma$ in (19) when $\delta=0$, but is greater when $\delta>0$. This is natural, since, with $\delta>0$, the bargaining game is more advantageous to the firm. In the limit as $\delta$ goes to 1 , the firm's share $\gamma$ also goes to 1 .

Using this lemma, the working paper version demonstrated that all the results in Propositions 2 and 3 hold for any $\delta \in[0,1)$ and employed this generalization to analyze the choice between integration and outsourcing. Briefly, suppose that for an integrated firm, we have $\delta>0$, while with outsourcing $\delta=0 .{ }^{29}$ Then, when the choice of the upfront payment $\tau$ is not restricted, it can be shown that the firm always prefers outsourcing to vertical integration. ${ }^{30}$ In contrast, when suppliers face credit constraints (in the sense that the upfront payment $\tau$ is restricted to be nonnegative), integration may be preferable to outsourcing. In particular, when credit constraints are present and $\alpha+\beta<1$, there exists $\bar{\delta} \in(0,1)$ such that vertical integration is preferred by the firm for $\delta<\bar{\delta}$ and outsourcing is preferred for $\delta>\bar{\delta}$. Furthermore, $\bar{\delta}$ is decreasing in $\alpha$, so that integration is more likely when there is greater complementarity between intermediate inputs. This result implies that vertical integration is more likely when both contractual frictions and credit market imperfections are present. ${ }^{31}$

\subsection{General Equilibrium}

We now discuss how the technology choice can be embedded in a general equilibrium version of our model. Besides verifying that general equilibrium interactions do not reverse our partial equilibrium results, this analysis is useful as a preparation for the results in the next subsection, where we investigate endogenous patterns of comparative advantage across countries. We start with an equilibrium with a given number of producers (final goods) and then endogenize this with free entry. In this and the next subsection, we focus on the case where $0<\mu<1$, and $\delta=0$ as in the baseline model.

Assume that there exists a continuum of final goods $q(z)$, with $z \in[0, Q]$, where $Q$ represents the number (measure) of final goods. All consumers have identical preferences,

$$
u=\left(\int_{0}^{Q} q(z)^{\beta} d z\right)^{1 / \beta}-c_{x} e, \quad 0<\beta<1,
$$

\footnotetext{
${ }^{29} \mathrm{An}$ integrated firm has $\delta>0$ because in this case the firm owns all the intermediate inputs. In this event the most a supplier can do is not to cooperate in the use of her intermediate input, which will reduce the efficiency with which the firm can employ this input, but may not reduce this efficiency to zero. See Grossman and Hart (1986).

${ }^{30}$ This is because the firm does not undertake any relationship-specific investments. Since suppliers are the only agents undertaking noncontractible relationship-specific investments, it is efficient to give them as much bargaining power as possible. This will not necessarily be the case if the firm were to also make relationship-specific investments. See, for example, Hart and Moore (1990), Antràs (2003, 2005), and Antràs and Helpman (2004).

${ }^{31}$ The positive effect of complementarity on the integration decision has been derived before in the property-rights literature (see Hart and Moore, 1990).
} 
where $e$ is the total effort exerted by this individual and the elasticity of substitution between final goods, $1 /(1-\beta)$, is greater than 1 , and $c_{x}$ represents the cost of effort in terms of real consumption. These preferences imply the demand function

$$
q(z)=\left[\frac{p(z)}{p^{I}}\right]^{-1 /(1-\beta)} \frac{S}{p^{I}},
$$

where $p(z)$ is the price of good $z, S$ is the aggregate spending level, and

$$
p^{I} \equiv\left[\int_{0}^{Q} p(z)^{-\beta /(1-\beta)} d z\right]^{-(1-\beta) / \beta}
$$

is the ideal price index, which we take to be the numeraire, i.e., $p^{I}=1$. The implied demand function $A[p(z)]^{-1 /(1-\beta)}$ for each firm is therefore identical to the demand function used in the previous sections, with $A=S$.

Recall that each firm in this economy solves the maximization problem (24) and its reducedform profit function is given by (29). We assume that the degree of technological complementarity, $\alpha$, varies across firms (or sectors) with its support given by a subset of $(0,1)$. We denote its cumulative distribution function by $H(\alpha)$. This formulation implies that if $Q$ products are available for consumption, a fraction $H(\alpha)$ of them are produced with elasticities of substitution smaller than $1 /(1-\alpha)$.

The key general equilibrium interaction results from competition of producers for a scarce resource, labor. Assume that labor is in fixed supply $L$. A firm that adopts technology $N$ employs $N$ individuals as suppliers and $C_{L}(N)$ workers in the process of technology adoption (implementation, use, or creation of the technology). We assume that these are the only uses of labor (Assumption 1 now applies to $\left.C_{L}(N)\right)$. Denoting the wage rate in terms of the numeraire by $w$, this implies that the total cost of adopting technology $N$ is $C(N)=w C_{L}(N)$. The wage rate $w$ is taken as given by each firm, but is endogenously determined in equilibrium.

The first-order condition of the maximization of (29) yields:

$$
\frac{\beta \kappa}{1-\beta} A Z(\alpha, \mu) N^{\frac{\beta(\kappa+1)-1}{1-\beta}}=w C_{L}^{\prime}(N)+w_{0}, \text { for all } \alpha \in(0,1)
$$

Since each individual can be employed at the wage $w$ in the process of technology adoption, their outside option as a supplier is $w_{0}=w$. Equation (34) then implies that the technology choice depends on $a \equiv A / w$, which is the inverse real cost of technology adoption (or the "equilibrium market size"). Let the equilibrium technology choice of $N$ as a function of $a, \alpha$, and $\mu$ implied by (34) be $N(a, \alpha, \mu)$. Defining total labor demand by a firm with technology $N$ as $C_{T}(N) \equiv C_{L}(N)+N$, condition (34) implies that $N(a, \alpha, \mu)$ is implicitly defined by

$$
\frac{\beta \kappa}{1-\beta} a Z(\alpha, \mu) N(a, \alpha, \mu)^{\frac{\beta(\kappa+1)-1}{1-\beta}}=C_{T}^{\prime}[N(a, \alpha, \mu)], \text { for all } \alpha \in(0,1) .
$$


Since firms with higher elasticities of substitution choose higher $N$ (Proposition 2), this equation implies that $N(a, \alpha, \mu)$ is increasing in $\alpha$. The demand for labor by a firm choosing technology $N(a, \alpha, \mu)$ is $C_{T}[N(a, \alpha, \mu)]$, thus labor market clearing can be expressed as: ${ }^{32}$

$$
Q \int_{0}^{1} C_{T}[N(a, \alpha, \mu)] d H(\alpha)=L
$$

where the left-hand side is total demand for labor and $L$ is labor supply. Since $N(a, \alpha, \mu)$ is increasing in $a$, this condition uniquely determines the equilibrium value of $a$, i.e., our measure of the real demand level. The relationship between $a$ and the model's parameters, as embodied in this equation, illustrates the key general equilibrium feedback in our model. Proposition 2 implies that $N(a, \alpha, \mu)$ is increasing in $\mu$, so we may expect better contracting institutions to encourage all firms to adopt more advanced technologies. However, the resource constraint, (36), implies that not all the $N(a, \alpha, \mu)$ 's can increase with $Q$ constant. Consequently, the equilibrium value of $a$ has to adjust to clear the labor market when $\mu$ rises.

To derive the implications of an increase in $\mu$ on the cross-sectional distribution of technology choices, recall that the number of products, $Q$, is given, and differentiate the first-order condition (35) to obtain

$$
\hat{a}+\zeta_{\mu}(\alpha, \mu) \hat{\mu}=\lambda[N(a, \alpha, \mu)] \hat{N}(a, \alpha, \mu),
$$

where $\hat{y}$, defined as $d y / y$, represents the proportional rate of change of variable $y, \zeta_{\mu}(\alpha, \mu)$ is the elasticity of $Z(\alpha, \mu)$ with respect to $\mu$, and $\lambda(N)$ is the elasticity of the marginal cost curve $C_{T}^{\prime}(N)$ minus $[\beta(\kappa+1)-1] /(1-\beta)$, i.e.,

$$
\lambda(N) \equiv \frac{C_{T}^{\prime \prime}(N) N}{C_{T}^{\prime}(N)}-\frac{\beta(\kappa+1)-1}{1-\beta} .
$$

Assumption 1 implies that $\lambda(N)>0$. Moreover, as proved in Lemma $2 \zeta_{\mu}(\alpha, \mu)>0$ and $\zeta_{\mu}(\alpha, \mu)$ is decreasing in $\alpha$. Next differentiating (36), we obtain a relationship between $\mu$ and $a$ :

$$
\int_{0}^{1} \sigma_{L}(\alpha) \hat{N}(a, \alpha, \mu) d H(\alpha)=0
$$

where $\sigma_{L}(\alpha) \equiv C_{T}^{\prime}[N(a, \alpha, \mu)] N(a, \alpha, \mu)$. Substituting (37) into (38) then yields

$$
\hat{a}=-\frac{\int_{0}^{1} \sigma_{L}(\alpha) \zeta_{\mu}(\alpha, \mu) \lambda[N(a, \alpha, \mu)]^{-1} d H(\alpha)}{\int_{0}^{1} \sigma_{L}(\alpha) \lambda[N(a, \alpha, \mu)]^{-1} d H(\alpha)} \hat{\mu} .
$$

Since the term in front of $\hat{\mu}$ on the right-hand side of this equation is negative, an improvement in contracting institutions increases wages relative to expenditure and reduces $a$.

Equation (38) shows that the proportional change in $N$ in response to $\hat{\mu}, \hat{N}(a, \alpha, \mu)$, can be positive only for some $\alpha$ 's (again because of the resource constraint). Since $\partial \zeta_{\mu}(\alpha, \mu) / \partial \alpha<0$

\footnotetext{
${ }^{32}$ Since it is straightforward to verify that the wage is always strictly positive, (36) is written as an equality rather than in complementary slackness form.
} 
(from Lemma 2), the left-hand side of equation (37) is decreasing in $\alpha$. Consequently, there exists a critical value $\alpha_{\mu}$ such that $\hat{N}(a, \alpha, \mu)>0$ for all $\alpha<\alpha_{\mu}$ and $\hat{N}(a, \alpha, \mu)<0$ for all $\alpha>\alpha_{\mu}$. This implies that low $\alpha$ firms, with greater technological complementarity between inputs, are more contract dependent. This establishes the following proposition (proof in the text):

Proposition 4 Suppose Assumption 1 holds. Then there exists $\alpha_{\mu} \in(0,1)$ such that in the general equilibrium economy with $Q$ constant, an increase in $\mu$ raises the level of technology $N(a, \alpha, \mu)$ in all firms with $\alpha<\alpha_{\mu}$ and reduces it in all firms with $\alpha>\alpha_{\mu}$.

We next discuss how the number of products, $Q$, can be endogenized with free entry. To do this in the simplest possible way, suppose that an entrant faces a fixed cost of entry $w f$, where $f$ is the amount of labor required for entry. This cost is borne in addition to the cost of technology adoption. Moreover, in the spirit of Hopenhayn (1992) and Melitz (2003), suppose that an entrant does not know a key parameter of the technology prior to entry. While in their models the entrant does not know its own productivity, we assume instead that it does not know $\alpha$, but knows that $\alpha$ is drawn from the cumulative distribution function $H(\alpha)$. Since the relationship between $\alpha$ and productivity is determined in general equilibrium, the distribution of productivity is endogenous.

After entry, each firm learns its $\alpha$ and maximizes the profit function (29). This maximization leads to the choice of technology as a function of $a, \mu$, and $\alpha$, i.e., $N(a, \alpha, \mu)$, in (35). Let us define

$$
\Pi(a, \alpha, \mu) \equiv a Z(\alpha, \mu) N(a, \alpha, \mu)^{1+\frac{\beta(\kappa+1)-1}{1-\beta}}-C_{T}[N(a, \alpha, \mu)],
$$

where $Z(\alpha, \mu)$ is given by (30). This is an indirect profit function, with profits measured in units of labor. It is straightforward to verify that it is increasing in $a, \alpha$ and $\mu$.

Free entry implies that expected profits must equal the entry cost $w f$, or

$$
\int_{0}^{1} \Pi(a, \alpha, \mu) d H(\alpha)=f
$$

This free entry condition uniquely determines the equilibrium value of $a$, without reference to the labor market clearing condition (36). Consequently, given the equilibrium value of $a$, the distribution of $\alpha$ induces a distribution of productivity and firm size in the economy (from the first-order condition (35)): firms with larger $\alpha$ 's choose more advanced technologies and they are more productive. ${ }^{33}$

Finally, the labor market clearing condition determines the number of entrants. Since labor demand now includes individuals working in the founding of the firms, the market clearing condition

\footnotetext{
${ }^{33}$ Note also that the degree of dispersion of productivity depends on the degree of contract incompleteness; in countries with better contracting institutions there is less productivity dispersion and less size dispersion. This follows from the fact that small firms (i.e., low- $\alpha$ firms) are larger in countries with better contracting institutions, while large firms (high- $\alpha$ firms) are smaller in those same countries. This prediction of the model is consistent with the empirical evidence reported in Tybout (2000), which indicates that there are significantly fewer medium-sized enterprises in less-developed economies, which typically have worse contracting institutions.
} 
(36) has to be replaced with

$$
Q\left[\int_{0}^{1} C_{T}[N(a, \alpha, \mu)] d H(\alpha)+f\right]=L
$$

Together with $N(a, \alpha, \mu)$ from (35) and $a$ from the free entry condition (39), this modified labor market clearing condition determinants the number of entrants $Q$.

An interesting implication of the general equilibrium with free entry is that an increase in the supply of labor $L$ does not create a scale effect and is not a source of comparative advantage. If two countries that differ only in $L$ freely trade with each other, their wages are equalized, their technology level is the same in every industry $\alpha$, and they have the same distribution of productivity and firm size. The only difference is that the larger country has proportionately more final good producers. This result contrasts with the case with an exogenous number of products, where differences in $L$ would generate comparative advantage. ${ }^{34}$

\subsection{Comparative Advantage}

Perhaps the most interesting general equilibrium application of our framework is to international trade. Consider a world consisting of two countries, indexed by $\ell=1,2$. We now derive an endogenous pattern of comparative advantage between these two countries from differences in contracting institutions. ${ }^{35}$ Suppose that there is a fixed number of products and that every product is distinct not only from other products produced in its own country, but also from products produced in the foreign country. All products can be freely traded between the two countries.

Suppose also that the two countries are identical, except for their contracting institutions. In particular, $L^{1}=L^{2}, Q^{1}=Q^{2}$ and $H^{1}(\alpha)=H^{2}(\alpha)$ for all $\alpha \in(0,1)$, but the fraction of activities $\mu^{\ell}$ that are contractible differs across countries. Without loss of generality, we assume that $\mu^{1}>\mu^{2}$, so that country 1 has better contracting institutions.

The equilibrium condition for technology adoption (34) holds in both countries, with different wage rates, $w^{\ell}$, for the two countries ( $A$ is the same for both countries and equal to world expenditure). Defining $a^{\ell} \equiv A / w^{\ell}$ and $N^{\ell}(\alpha) \equiv N\left(a^{\ell}, \alpha, \mu^{\ell}\right)$, we have

$$
\frac{\beta \kappa}{1-\beta} a^{\ell} Z\left(\alpha, \mu^{\ell}\right) N^{\ell}(\alpha)^{\frac{\beta(\kappa+1)-1}{1-\beta}}=C_{T}^{\prime}\left[N^{\ell}(\alpha)\right], \text { for all } \alpha \in(0,1), \quad \ell=1,2 .
$$

In addition, the labor market clearing condition (36) holds in both countries. Consequently, the country with better contracting institutions, country 1 , will have higher wages and a lower $a^{\ell}{ }^{36}$

The pattern of trade can now be determined by comparing the revenues of firms with the same value of $\alpha$ in the two countries. We show in the Appendix (see the proof of Proposition 2) that the

\footnotetext{
${ }^{34}$ We have also worked out an endogenous growth model with expanding product variety, where the long-run rate of growth depends on the degree of contract incompleteness. We do not discuss this model in order to save space.

${ }^{35}$ The link between contracting institutions and endogenous comparative advantage was previously discussed by Levchenko (2003), Costinot (2004), Nunn (2005) and Antràs (2005).

${ }^{36}$ Suppose not, then (40) implies $N^{1}(\alpha)>N^{2}(\alpha)$ for all $\alpha$ 's (since $N^{\ell}(\alpha)$ is increasing in $\mu$ for given $a$ ), and so labor market clearing cannot be satisfied in both countries.
} 
revenue of a producer with parameter $\alpha$ in country $\ell$ is

$$
R^{\ell}(\alpha)=A Z\left(\alpha, \mu^{\ell}\right) N^{\ell}(\alpha)^{\frac{\beta \kappa}{1-\beta}} \frac{1-\beta\left(1-\mu^{\ell}\right)}{(1-\beta)\left[1-\beta\left(1-\mu^{\ell}\right) \frac{\alpha}{\alpha+\beta}\right]},
$$

which is increasing in total world expenditure, $A$, in $Z(\alpha, \mu)$, and in the level of technology. But it is also directly affected by the parameters through the last term on the right-hand side.

If $R^{1}(\alpha) / R^{2}(\alpha)>\int_{0}^{1} R^{1}(\alpha) d H(\alpha) / \int_{0}^{1} R^{2}(\alpha) d H(\alpha)$, then country 1 is a net exporter of goods with substitution parameter $\alpha$. Consequently, we simply need to determine the distribution of $R^{1}(\alpha) / R^{2}(\alpha)$ across different $\alpha$ 's. > From (41) we have

$$
\frac{R^{1}(\alpha)}{R^{2}(\alpha)}=\frac{Z\left(\alpha, \mu^{1}\right)}{Z\left(\alpha, \mu^{2}\right)}\left[\frac{N^{1}(\alpha)}{N^{2}(\alpha)}\right]^{\frac{\beta \kappa}{1-\beta}} \frac{1-\beta\left(1-\mu^{1}\right)}{1-\beta\left(1-\mu^{2}\right)} \frac{1-\beta\left(1-\mu^{2}\right) \frac{\alpha}{\alpha+\beta}}{1-\beta\left(1-\mu^{1}\right) \frac{\alpha}{\alpha+\beta}} .
$$

Both $Z\left(\alpha, \mu^{1}\right) / Z\left(\alpha, \mu^{2}\right)$ and the last term in (42) are decreasing in $\alpha{ }^{37}$ Second, Proposition 4 implies that there exists an $\alpha_{\mu}$ such that $N^{1}(\alpha)<N^{2}(\alpha)$ for all $\alpha>\alpha_{\mu}$ and $N^{1}(\alpha)>N^{2}(\alpha)$ for all $\alpha<\alpha_{\mu}$. As a result, country 1 , which has the better contracting institutions, tends to export low- $\alpha$ products and import high- $\alpha$ products. If, in addition, $\lambda(N)$ is constant, the proportional change in $N(a, \alpha, \mu)$ in response to an increase in $\mu$ is always smaller when $\alpha$ is greater (because the partial $\partial \zeta_{\mu}(\alpha, \mu) / \partial \alpha$ is negative, see Lemma 2). In this case, $R^{1}(\alpha) / R^{2}(\alpha)$ is everywhere decreasing in $\alpha$ and there exists $\bar{\alpha}_{\mu} \in(0,1)$ such that $R^{1}(\alpha) / R^{2}(\alpha)>\int_{0}^{1} R^{1}(\alpha) d H(\alpha) / \int_{0}^{1} R^{2}(\alpha) d H(\alpha)$ for all $\alpha<\bar{\alpha}_{\mu}$ and the opposite inequality holds for all $\alpha>\bar{\alpha}_{\mu}$. Consequently, country 1 , with the better contracting institutions, is a net exporter in low- $\alpha$ sectors and a net importer in high- $\alpha$ sectors. This result is summarized in the following (proof in the text):

Proposition 5 Suppose Assumption 1 holds and $\lambda(N)$ is constant. Then there exists $\bar{\alpha}_{\mu} \in(0,1)$ such that in the two-country world equilibrium country 1 with $\mu^{1}>\mu^{2}$ is a net exporter of products with $\alpha<\bar{\alpha}_{\mu}$ and a net importer of products with $\alpha>\bar{\alpha}_{\mu}$.

The most important implication of this result is that differences in contracting institutions create endogenous comparative advantage. A country with better contracting institutions gains a comparative advantage in sectors that are more contract dependent, which, in our model, correspond to the sectors with greater technological complementarities between inputs.

The main implications in Proposition 5 receive support from a number of recent empirical papers. Nunn (2005) and Levchenko (2003) use international trade data and the classification of industries into more and less "contract dependent" groups. Consistent with Proposition 5, they find that countries with better contracting institutions specialize in the export of goods that are more "contract dependent". Moreover, the impact of cross-country differences in the contracting institutions on exports is quantitative large. Nunn, for example, finds that the estimated impact

\footnotetext{
${ }^{37}$ Recall from Lemma 2 that $Z(\alpha, \mu)$ is increasing in $\alpha$ and $\mu$, and $\partial \zeta_{\alpha}(\alpha, \mu) / \partial \mu<0$. Therefore $Z\left(\alpha, \mu^{1}\right) / Z\left(\alpha, \mu^{2}\right)$ is decreasing in $\alpha$ whenever $\mu^{1}>\mu^{2}$. The fact that the last term is decreasing in $\alpha$ follows from $\mu^{1}>\mu^{2}$.
} 
of the quality of the legal system on the export of contract-dependent sectors is comparable to the effect of human capital abundance on the export of human capital intensive products. In other

words, according to these estimates, differences in legal systems are as important as differences in human capital abundance for explaining trade flows.

\section{Conclusion}

In this paper, we developed a tractable framework for the analysis of the impact of contractual incompleteness and technological complementarities on the equilibrium technology choice. In our model, a firm chooses its technology corresponding to the range of intermediate inputs used in production, and offers contracts to suppliers, specifying the required investments in contractible activities. Investments in the remaining, noncontractible activities are then chosen by the suppliers in anticipation of the ex post bargaining payoffs.

We used the Shapley value to characterize the division of surplus between the firm and its suppliers, and derived an explicit solution to these payoffs. Using this setup, we established that greater contractual incompleteness reduces investments in noncontractible and contractible activities and depresses technology choice. The impact of contractual incompleteness on technology adoption is greater in sectors with more complementary intermediate inputs.

The key mechanism developed in the paper leads to potentially large differences in productivity in response to differences in contracting institutions. We also derived implications of this mechanism for differences in the internal organization of the firm across societies with different contracting institutions and a new mechanism for endogenous comparative advantage. These implications are consistent with a range of recent empirical results in the literature.

It is also useful to note that while we have used a "love-for-variety" model of technology, our results do not depend on this specific modeling approach. In equation (29) $N$ can be interpreted as a general technological investment, and the linkages between contracts and technology adoption (or investment) highlighted in our analysis would apply independent from the specific assumptions regarding the nature of technology.

A number of areas are left for future research. These include, but are not limited to, the following. The model assumes that all activities are symmetric; an important extension is to see whether similar results hold with a more general production function, where the firm may wish to treat some suppliers of intermediate inputs differently than others, depending, for example, on how essential they are for production. Another area for future study is an investigation of the simultaneous determination of the range of intermediate inputs used by the firm and the division of labor among the suppliers. Finally, it is important to investigate whether the relationship between contracting institutions, technological complementarities, and the choice of technology, is fundamentally different when we use alternative approaches to the theory of the firm, such as the managerial incentives approach of Holmström and Milgrom (1991). 


\section{Appendix}

\section{Second-Order Conditions in the Complete Contracting Case}

Let $\Pi=A^{1-\beta} N^{\beta(\kappa+1)} x^{\beta}-c_{x} N x-C(N)-w_{0} N$. Using the first-order conditions (11) and (12), the matrix of the second-order conditions can be expressed as:

$$
\left(\begin{array}{cc}
\partial^{2} \Pi / \partial x^{2} & \partial^{2} \Pi / \partial x \partial N \\
\partial^{2} \Pi / \partial N \partial x & \partial^{2} \Pi / \partial N^{2}
\end{array}\right)=\left(\begin{array}{cc}
-(1-\beta) \kappa N c_{x}^{2}\left(C^{\prime}+w_{0}\right)^{-1} & -c_{x}[1-\beta(1+\kappa)] \\
-c_{x}[1-\beta(1+\kappa)] & -N^{-1}[1-\beta(1+\kappa)](\kappa+1) \kappa^{-1}\left(C^{\prime}+w_{0}\right)-C^{\prime \prime}
\end{array}\right)
$$

The second-order conditions are satisfied if this matrix is negative definite, which requires its diagonal elements to be negative and its determinant to be positive. The first diagonal element is negative; the second diagonal element is negative if and only if

$$
\frac{C^{\prime \prime} N}{C^{\prime}+w_{0}}>\frac{[\beta(1+\kappa)-1](\kappa+1)}{\kappa},
$$

and the determinant is positive if and only if

$$
\frac{C^{\prime \prime} N}{C^{\prime}+w_{0}}>\frac{\beta(1+\kappa)-1}{1-\beta} .
$$

Note that if $\beta(1+\kappa)<1$ both of these conditions are satisfied, and if $\beta(1+\kappa) \geq 1$, the second inequality implies the first inequality. Therefore Assumption 1 is necessary and sufficient for the second-order conditions to be satisfied.

\section{Proof of Proposition 1}

The first part of the proposition is a direct implication of Assumption 1. The comparative statics of $N^{*}$ follow from the implicit function theorem by noting that, except for $\beta, N^{*}$ increases in response to an increase in a parameter if and only if this parameter raises the left-hand side of (11). Using the results concerning the response of $N^{*}$ to changes in parameters together with (12) then implies the responses of $x^{*}$ to parameter changes.

\section{Proof of Lemma 1 and Related Results}

We develop here a more formal proof than the one in the main text that builds on the work of Aumann and Shapley (1974). Let there be $M$ suppliers each one controlling a range $\varepsilon=N / M$ of the continuum of intermediate inputs. Due to symmetry, all suppliers provide an amount $x_{c}$ of contractible activities. As for the noncontractible activities, consider a situation in which a supplier $j$ supplies an amount $x_{n}(j)$ per noncontractible activity, while the $M-1$ remaining suppliers supply the same amount $x_{n}(-j)$ (note that we are again appealing to symmetry).

To compute the Shapley value for this particular supplier $j$, we need to determine the marginal contribution of this supplier to a given coalition of agents. A coalition of $n$ suppliers and the firm yields a sales revenue of

$$
F_{I N}(n, N ; \varepsilon)=A^{1-\beta} N^{\beta(\kappa+1-1 / \alpha)} x_{c}^{\beta \mu}\left[(n-1) \varepsilon x_{n}(-j)^{(1-\mu) \alpha}+\varepsilon x_{n}(j)^{(1-\mu) \alpha}\right]^{\beta / \alpha},
$$


when the supplier $j$ is in the coalition, and a sales revenue

$$
F_{\text {OUT }}(n, N ; \varepsilon)=A^{1-\beta} N^{\beta(\kappa+1-1 / \alpha)} x_{c}^{\beta \mu}\left[n \varepsilon x_{n}(-j)^{(1-\mu) \alpha}\right]^{\beta / \alpha}
$$

when supplier $j$ is not in the coalition. Notice that even when $n<N$, the term $N^{\beta(\kappa+1-1 / \alpha)}$ remains in front, because it represents a feature of the technology, though productivity suffers because the term in square brackets is lower.

Following the notation in the main text, the Shapley value of player $j$ is

$$
s_{j}=\frac{1}{(M+1) !} \sum_{g \in G}\left[v\left(z_{g}^{j} \cup j\right)-v\left(z_{g}^{j}\right)\right] .
$$

The fraction of permutations in which $g(j)=i$ is $1 /(M+1)$ for every $i$. If $g(j)=0$ then $v\left(z_{g}^{j} \cup j\right)=$ $v\left(z_{g}^{j}\right)=0$, because in this event the firm is necessarily ordered after $j$. If $g(j)=1$ then the firm is ordered before $j$ with probability $1 / M$ and after $j$ with probability $1-1 / M$. In the former case $v\left(z_{g}^{j} \cup j\right)=F_{I N}(1, N ; \varepsilon)$, while in the latter case $v\left(z_{g}^{j} \cup j\right)=0$. Therefore the conditional expected value of $v\left(z_{g}^{j} \cup j\right)$, given $g(j)=1$, is $\frac{1}{M} F_{I N}(1, N ; \varepsilon)$. By similar reasoning, the conditional expected value of $v\left(z_{g}^{j}\right)$ is $\frac{1}{M} F_{O U T}(0, N ; \varepsilon)$. Repeating the same argument for $g(j)=i, i>1$, the conditional expected value of $v\left(z_{g}^{j} \cup j\right)$, given $g(j)=i$, is $\frac{i}{M} F_{I N}(i, N ; \varepsilon)$, and the conditional expected value of $v\left(z_{g}^{j}\right)$ is $\frac{i}{M} F_{O U T}(i-1, N ; \varepsilon)$. It follows from (A3) that

$$
\begin{aligned}
s_{j} & =\frac{1}{(M+1) M} \sum_{i=1}^{M} i\left[F_{I N}(i, N ; \varepsilon)-F_{O U T}(i-1, N ; \varepsilon)\right] \\
& =\frac{1}{(N+\varepsilon) N} \sum_{i=1}^{M} i \varepsilon\left[F_{I N}(i, N ; \varepsilon)-F_{O U T}(i-1, N ; \varepsilon)\right] \varepsilon
\end{aligned}
$$

Substituting for (A1) and (A2),

$$
\begin{aligned}
s_{j}= & \frac{A^{1-\beta} N^{\beta(\kappa+1-1 / \alpha)} x_{c}^{\beta \mu}}{(N+\varepsilon) N} \sum_{i=1}^{M} i \varepsilon\left\{i \varepsilon x_{n}(-j)^{(1-\mu) \alpha}+\varepsilon\left[x_{n}(j)^{(1-\mu) \alpha}-x_{n}(-j)^{(1-\mu) \alpha}\right]\right\}^{\beta / \alpha} \varepsilon \\
& -\frac{A^{1-\beta} N^{\beta(\kappa+1-1 / \alpha)} x_{c}^{\beta \mu}}{(N+\varepsilon) N} \sum_{i=1}^{M} i \varepsilon\left[i \varepsilon x_{n}(-j)^{(1-\mu) \alpha}-\varepsilon x_{n}(-j)^{(1-\mu) \alpha}\right]^{\beta / \alpha} \varepsilon .
\end{aligned}
$$

For $\varepsilon$ small enough, the first-order Taylor expansion gives

$$
s_{j} \simeq \frac{A^{1-\beta} N^{\beta(\kappa+1-1 / \alpha)} x_{c}^{\beta \mu}(\beta / \alpha) \varepsilon x_{n}(j)^{(1-\mu) \alpha}}{(N+\varepsilon) N} \sum_{i=1}^{M}(i \varepsilon)\left[i \varepsilon x_{n}(-j)^{(1-\mu) \alpha}\right]^{(\beta-\alpha) / \alpha} \varepsilon,
$$

or

$$
\frac{s_{j}}{\varepsilon} \simeq \frac{A^{1-\beta} N^{\beta(\kappa+1-1 / \alpha)}(\beta / \alpha)\left[\frac{x_{n}(j)}{x_{n}(-j)}\right]^{(1-\mu) \alpha} x_{c}^{\beta \mu} x_{n}(-j)^{\beta(1-\mu)}}{(N+\varepsilon) N} \sum_{i=1}^{M}(i \varepsilon)^{\beta / \alpha} \varepsilon .
$$

Now taking the limit as $M \rightarrow \infty$, and therefore $\varepsilon=N / M \rightarrow 0$, the sum on the right-hand side of this 
equation becomes a Riemann integral:

$$
\lim _{M \rightarrow \infty}\left(\frac{s_{j}}{\varepsilon}\right)=\frac{A^{1-\beta} N^{\beta(\kappa+1-1 / \alpha)}(\beta / \alpha)\left[\frac{x_{n}(j)}{x_{n}(-j)}\right]^{(1-\mu) \alpha} x_{c}^{\beta \mu} x_{n}(-j)^{\beta(1-\mu)}}{N^{2}} \int_{0}^{N} z^{\beta / \alpha} d z .
$$

Solving the integral delivers

$$
\lim _{M \rightarrow \infty}\left(s_{j} / \varepsilon\right)=(1-\gamma) A^{1-\beta}\left[\frac{x_{n}(j)}{x_{n}(-j)}\right]^{(1-\mu) \alpha} x_{c}^{\beta \mu} x_{n}(-j)^{\beta(1-\mu)} N^{\beta(\kappa+1)-1},
$$

with $\gamma=\alpha /(\alpha+\beta)$. This corresponds to equation (20) in the main text, and completes the proof of the lemma.

In addition, imposing symmetry, i.e., $x_{n}(j)=x_{n}(-j)$, the firm's payoff is

$$
s_{0}=A^{1-\beta} N^{\beta(\kappa+1)} x_{c}^{\beta \mu} x_{n}^{\beta(1-\mu)}-N s_{j}=\gamma A^{1-\beta} x_{c}^{\beta \mu} x_{n}^{\beta(1-\mu)} N^{\beta(\kappa+1)},
$$

as stated in equation (22) in the text.

\section{Proof of Proposition 2}

First, we verify that the second-order conditions are again satisfied under Assumption 1. To see this, note that the problem in (14) is strictly concave and delivers a unique $x_{n}=\bar{x}_{n}\left(N, x_{c}\right)$, as given in (23). Plugging this expression into (24) we obtain

$$
\pi=A^{\frac{1-\beta}{1-\beta(1-\mu)}} \Psi N^{1+\frac{\beta(\kappa+1)-1}{1-\beta(1-\mu)}} x_{c}^{\beta \mu /[1-\beta(1-\mu)]}-c_{x} x_{c} N \mu-C(N)-w_{0} N
$$

where

$$
\Psi \equiv\left[\left(c_{x}\right)^{-1} \alpha(1-\gamma)\right]^{\beta(1-\mu) /[1-\beta(1-\mu)]}[1-\alpha(1-\mu)(1-\gamma)] .
$$

The second-order conditions can be checked, analogously to the case with complete contracts, by computing the Hessian and checking that it is negative definite.

Here, we present an alternative proof which also serves to illustrate how the reduced-form profit function (29) in the main text is derived. In particular, notice that for a given level of $N$, the problem of choosing $x_{c}$ is convex (since $\beta \mu<1-\beta+\beta \mu$ ) and delivers a unique solution:

$$
x_{c}=A\left[\frac{\beta c_{x}^{-1} \Psi}{1-\beta(1-\mu)}\right]^{[1-\beta(1-\mu)] /(1-\beta)} N^{\frac{\beta(\kappa+1)-1}{1-\beta}} .
$$

Plugging this solution in (A4) then delivers

$$
\pi=A Z N^{1+\frac{\beta(\kappa+1)-1}{1-\beta}}-C(N)-w_{0} N
$$

where

$$
Z \equiv\left[\frac{\beta c_{x}^{-1}}{1-\beta(1-\mu)}\right]^{\beta \mu /(1-\beta)} \Psi^{[1-\beta(1-\mu)] /(1-\beta)}\left(\frac{1-\beta}{1-\beta(1-\mu)}\right)
$$

which simplifies to equation (30) in the text. This reduced-form expression of the profit function immediately implies that $\partial^{2} \Pi / \partial N^{2}<0$ if and only if the second part of Assumption 1 holds. Finally, from equation (A5) we also have $c_{x} x_{c} N \mu=A Z N^{1+\frac{\beta(\kappa+1)-1}{1-\beta}} \beta \mu /(1-\beta)$, which combined with (19) and (28) implies that 
the firm's revenues are

$$
\begin{aligned}
R & =\pi+C(N)+w_{0} N+c_{x} x_{c} N \mu+c_{x} x_{n} N(1-\mu) \\
& =A Z N^{1+\frac{\beta(\kappa+1)-1}{1-\beta}}\left(\frac{1-\beta(1-\mu)}{(1-\beta)\left[1-\beta(1-\mu) \frac{\alpha}{\alpha+\beta}\right]}\right),
\end{aligned}
$$

which will be used in Section 5.3 (see equation (41)).

Next, the comparative static results follow from the implicit function theorem as in the proof of Proposition 1. First, $\partial \tilde{N} / \partial A>0$ follows immediately. To show that $\partial \tilde{N} / \partial \alpha>0$ and $\partial \tilde{N} / \partial \mu>0$, let us take logarithms of both sides of (25), to obtain

$$
\frac{\beta(\kappa+1)-1}{1-\beta} \ln \tilde{N}+\ln \left(A \kappa \beta^{\frac{1}{1-\beta}} c_{x}^{-\frac{\beta}{1-\beta}}\right)+F(\vartheta, \mu)=\ln \left(C^{\prime}(\tilde{N})+w_{0}\right)
$$

where

$$
F(\vartheta, \mu)=\frac{1-\beta(1-\mu)}{1-\beta} \ln \left(\frac{1-\vartheta(1-\mu)}{1-\beta(1-\mu)}\right)+\frac{\beta(1-\mu)}{1-\beta} \ln \left(\vartheta \beta^{-1}\right),
$$

and $\vartheta \equiv \alpha(1-\gamma)<\beta$ is monotonically increasing in $\alpha$. Simple differentiation delivers

$$
\frac{\partial F(\vartheta, \mu)}{\partial \vartheta}=\frac{(1-\mu)(\beta-\vartheta)}{(1-\beta) \vartheta(1-\vartheta(1-\mu))}
$$

which implies $\partial F / \partial \alpha>0$, and establishes that $\partial \tilde{N} / \partial \alpha>0$.

Furthermore,

$$
\frac{\partial F(\vartheta, \mu)}{\partial \mu}=\frac{\vartheta-\beta+\beta(1-\vartheta(1-\mu))\left(\ln \left(\frac{1-\vartheta(1-\mu)}{1-\beta(1-\mu)}\right)+\ln \left(\frac{\beta}{\vartheta}\right)\right)}{(1-\vartheta(1-\mu))(1-\beta)}
$$

and

$$
\frac{\partial^{2} F(\vartheta, \mu)}{\partial \mu^{2}}=-\frac{(\beta-\vartheta)^{2}}{(1-\vartheta(1-\mu))^{2}(1-\beta(1-\mu))(1-\beta)}<0 .
$$

Thus, $\partial F(\vartheta, \mu) / \partial \mu$ reaches its minimum over the set $\mu \in[0,1]$ at $\mu=1$, in which case it equals

$$
\frac{\vartheta-\beta+\beta \ln (\beta / \vartheta)}{1-\beta}>0
$$

This inequality follows from the fact that $\vartheta-\beta+\beta \ln (\beta / \vartheta)$ is decreasing in $\vartheta$ for $\vartheta<\beta$, and is equal to 0 at $\vartheta=\beta$. We thus have shown that $\partial F(\vartheta, \mu) / \partial \mu>0$ for all $\mu$, so that $\partial \tilde{N} / \partial \mu>0$.

Finally, note that straightforward differentiation of (28) delivers $\partial\left(\tilde{x}_{n} / \tilde{x}_{c}\right) / \partial A=0, \partial\left(\tilde{x}_{n} / \tilde{x}_{c}\right) / \partial \alpha>0$ and $\partial\left(\tilde{x}_{n} / \tilde{x}_{c}\right) / \partial \mu>0$. And, in light of equation (26), the effects of $A, \alpha$ and $\mu$ on $\tilde{x}_{c}$ follow directly from those on $\tilde{N}$, where the inequalities become strict whenever $C^{\prime \prime}(\cdot)>0$.

\section{Proof of Lemma 2}

Comparison of (30) with (A6) implies that

$$
Z(\alpha, \mu) \equiv \varphi e^{F(\vartheta, \mu)},
$$


where

$$
\vartheta \equiv \alpha(1-\gamma)=\frac{\alpha \beta}{\alpha+\beta}
$$

and $\varphi$ is a parameter that only depends on $\beta$ and $c_{x}$. We have that $F(\vartheta, \mu)$ is increasing in both its arguments, and that $\vartheta$ is increasing in $\alpha$, which establish part 1 of the lemma. To prove part 2 , first note that

$$
\frac{\partial^{2} F(\vartheta, \mu)}{\partial \vartheta \partial \mu}=\frac{-(\beta-\vartheta)}{(1-\vartheta(1-\mu))^{2} \vartheta(1-\beta)}<0,
$$

because $\vartheta<\beta$. Moreover,

$$
\zeta_{\mu}(\alpha, \mu) \equiv \frac{\mu}{Z} \frac{\partial Z(\alpha, \mu)}{\partial \mu}=\mu \frac{\partial F(\vartheta, \mu)}{\partial \mu},
$$

and therefore

$$
\frac{\partial \zeta_{\mu}(\alpha, \mu)}{\partial \alpha}=\mu \frac{\partial^{2} F(\vartheta, \mu)}{\partial \vartheta \partial \mu} \times \frac{\partial \vartheta}{\partial \alpha}<0 .
$$

Similarly,

$$
\zeta_{\alpha}(\alpha, \mu) \equiv \frac{\alpha}{Z} \frac{\partial Z(\alpha, \mu)}{\partial \alpha}=\alpha \frac{\partial F(\vartheta, \mu)}{\partial \vartheta} \times \frac{\partial \vartheta}{\partial \alpha},
$$

and therefore

$$
\frac{\partial \zeta_{\alpha}(\alpha, \mu)}{\partial \mu}=\alpha \frac{\partial^{2} F(\vartheta, \mu)}{\partial \vartheta \partial \mu} \times \frac{\partial \vartheta}{\partial \alpha}<0 .
$$

\section{Proof of Proposition 3}

The proof follows from Proposition 2 , since $\left\{\tilde{N}, \tilde{x}_{c}\right\}$ converge to $\left\{N^{*}, x^{*}\right\}$ as $\mu \rightarrow 1$, and $\tilde{N}, \tilde{x}_{c}$, and $\tilde{x}_{n}$ are all increasing in $\mu$ (nondecreasing in $\mu$, in the case of the investment levels).

\section{Proof of Lemma 3}

The proof is similar to that of Lemma 1. A coalition of $n$ suppliers and the firm yields a sales revenue of

$F_{I N}(n, N ; \varepsilon)=A^{1-\beta} N^{\beta(\kappa+1-1 / \alpha)} x_{c}^{\beta \mu}\left[(n-1) \varepsilon x_{n}(-j)^{(1-\mu) \alpha}+\varepsilon x_{n}(j)^{(1-\mu) \alpha}+(N-n \varepsilon) \delta^{\alpha} x_{n}(-j)^{(1-\mu) \alpha}\right]^{\beta / \alpha}$,

when the supplier $j$ is in the coalition, and a sales revenue

$F_{\text {OUT }}(n, N ; \varepsilon)=A^{1-\beta} N^{\beta(\kappa+1-1 / \alpha)} x_{c}^{\beta \mu}\left[n \varepsilon x_{n}(-j)^{(1-\mu) \alpha}+\varepsilon \delta^{\alpha} x_{n}(j)^{(1-\mu) \alpha}+(N-(n+1) \varepsilon) \delta^{\alpha} x_{n}(-j)^{(1-\mu) \alpha}\right]^{\beta / \alpha}$

when supplier $j$ is not in the coalition.

As in the case with $\delta=0$, the Shapley value of supplier $j$ can be written as

$$
s_{j}=\frac{1}{(N+\varepsilon) N} \sum_{i=1}^{M} i \varepsilon\left[F_{I N}(i, N ; \varepsilon)-F_{O U T}(i-1, N ; \varepsilon)\right] \varepsilon .
$$

Plugging the new formulas for $F_{I N}(n, N ; \varepsilon)$ and $F_{O U T}(n, N ; \varepsilon)$ in $(\mathrm{A} 7)$ and $(\mathrm{A} 8)$ then delivers 


$$
\begin{aligned}
s_{j}= & \frac{A^{1-\beta} N^{\beta(\kappa+1-1 / \alpha)} x_{c}^{\beta \mu}}{(N+\varepsilon) N} \sum_{i=1}^{M} i \varepsilon\left\{\begin{array}{c}
i \varepsilon\left(1-\delta^{\alpha}\right) x_{n}(-j)^{(1-\mu) \alpha}+\varepsilon\left[x_{n}(j)^{(1-\mu) \alpha}-x_{n}(-j)^{(1-\mu) \alpha}\right] \\
+N \delta^{\alpha} x_{n}(-j)^{(1-\mu) \alpha}
\end{array}\right\}^{\beta / \alpha} \varepsilon \\
& -\frac{A^{1-\beta} N^{\beta(\kappa+1-1 / \alpha)} x_{c}^{\beta \mu}}{(N+\varepsilon) N} \sum_{i=1}^{M} i \varepsilon\left[\begin{array}{c}
i \varepsilon\left(1-\delta^{\alpha}\right) x_{n}(-j)^{(1-\mu) \alpha}+\varepsilon\left[\delta^{\alpha} x_{n}(j)^{(1-\mu) \alpha}-x_{n}(-j)^{(1-\mu) \alpha}\right. \\
+N \delta^{\alpha} x_{n}(-j)^{(1-\mu) \alpha}
\end{array}\right]^{\beta / \alpha} \varepsilon .
\end{aligned}
$$

For $\varepsilon$ small enough, the first-order Taylor expansion gives

$$
\frac{s_{j}}{\varepsilon} \simeq \frac{A^{1-\beta} N^{\beta(\kappa+1-1 / \alpha)}(\beta / \alpha)\left(1-\delta^{\alpha}\right)\left[\frac{x_{n}(j)}{x_{n}(-j)}\right]^{(1-\mu) \alpha} x_{c}^{\beta \mu} x_{n}(-j)^{\beta(1-\mu)}}{(N+\varepsilon) N} \sum_{i=1}^{M}(i \varepsilon)\left[(i \varepsilon)\left(1-\delta^{\alpha}\right)+N \delta^{\alpha}\right]^{(\beta-\alpha) / \alpha} \varepsilon .
$$

Taking the limit as $M \rightarrow \infty$, now yields

$$
\lim _{M \rightarrow \infty}\left(\frac{s_{j}}{\varepsilon}\right)=\frac{A^{1-\beta} N^{\beta(\kappa+1-1 / \alpha)}(\beta / \alpha)\left(1-\delta^{\alpha}\right)\left[\frac{x_{n}(j)}{x_{n}(-j)}\right]^{(1-\mu) \alpha} x_{c}^{\beta \mu} x_{n}(-j)^{\beta(1-\mu)}}{N^{2}} \int_{0}^{N} z\left[z\left(1-\delta^{\alpha}\right)+N \delta^{\alpha}\right]^{(\beta-\alpha) / \alpha} d z .
$$

Finally, integrating by parts delivers

$$
\lim _{M \rightarrow \infty}\left(s_{j} / \varepsilon\right)=(1-\gamma) A^{1-\beta}\left[\frac{x_{n}(j)}{x_{n}(-j)}\right]^{(1-\mu) \alpha} x_{c}^{\beta \mu} x_{n}(-j)^{\beta(1-\mu)} N^{\beta(\kappa+1)-1},
$$

where

$$
\gamma \equiv \frac{\alpha\left(1-\delta^{\alpha+\beta}\right)}{(\alpha+\beta)\left(1-\delta^{\alpha}\right)}
$$

as claimed in the Lemma. 


\section{References}

Acemoglu, Daron, Pol Antràs, and Elhanan Helpman (2005), "Contracts and the Division of Labor," NBER Working Paper No. 11356.

Acemoglu, Daron and Fabrizio Zilibotti (1999), "Information Accumulation in Development," Journal of Economic Growth, 4, pp. 5-38.

Amaral, Pedro S. and Erwan Quintin (2005), "Finance Matters," mimeo.

Antràs, Pol (2003), "Firms, Contracts, and Trade Structure," Quarterly Journal of Economics, 118:4, pp. 1375-1418.

Antràs, Pol (2005), "Incomplete Contracts and the Product Cycle," American Economic Review, 95:4, pp. 1054-1073.

Antràs, Pol and Elhanan Helpman (2004), "Global Sourcing," Journal of Political Economy 112:3, pp. 552-580.

Aumann, Robert J. and Lloyd S. Shapley (1974), Values of Non-Atomic Games, Princeton University Press, Princeton, NJ.

Barro, Robert J., and Xavier Sala-i-Martin (2003), Economic Growth, Second Edition, Cambridge: MIT Press.

Bakos, Yannis and Erik Brynjolfsson (1993), "From Vendors to Partners: Information Technology and Incomplete Contracts in Buyer-supplier Relationships," Journal of Organizational Computing 3:3, pp. 301-328.

Basu, Susanto (1995), "Intermediate Goods and Business Cycles: Implications for Productivity and Welfare," American Economic Review 85:3, pp. 512-531.

Becker, Gary S. and Kevin M. Murphy (1992), "The Division of Labor, Coordination Costs, and Knowledge," Quarterly Journal of Economics, 107:4, pp. 1137-1160.

Benassy, Jean-Pascal (1998), "Is there always too little research in endogenous growth with expanding product variety?," European Economic Review, 42:1, pp. 61-69.

Bils, Mark and Peter J. Klenow (2001), "The Acceleration in Variety Growth," American Economic Review (Papers and Proceedings), 91:2, pp. 274-280.

Blanchard, Olivier and Michael Kremer (1997), "Disorganization," Quarterly Journal of Economics, Vol. 112:4, pp. 1091-1126.

Broda, Christian and David E. Weinstein (1996), "Globalization and the Gains from Variety," Quarterly Journal of Economics CXXI:2, pp.541-585. 
Caselli, Francesco (2004), "Accounting for Cross-Country Income Differences," forthcoming in the Handbook of Economic Growth.

Castro, Rui, Gian Luca Clementi and Glenn MacDonald (2004), "Investor Protection, Optimal Incentives, and Economic Growth," Quarterly Journal of Economics CXIX:3, pp. 1131-1175.

Costinot, Arnaud (2004), "Contract Enforcement, Division of Labor and the Pattern of Trade," manuscript Princeton University.

Erosa, Andrés and Ana Hidalgo Cabrillana (2005), "On Capital Market Imperfections as a Source of Low TFP and Economic Rents," mimeo.

Ethier, Wilfred J (1982), "National and International Returns to Scale in the Modern Theory of International Trade," American Economic Review, 72:3, pp. 389-405.

de Fontenay, Catherine C. and Joshua S. Gans (2003), "Organizational Design and Technology Choice under Intrafirm Bargaining: Comment," American Economic Review, 93:1, pp. 448455.

Francois, Patrick and Joanne Roberts (2003), "Contracting Productivity Growth," Review of Economic Studies, 70, pp. 59-85.

Grether, Jean-Marie (1996), "Mexico, 1985-90: Trade Liberalization, Market Structure, and Manufacturing Performance," in Mark J. Roberts and James R. Tybout (eds.) (1996) Industrial Evolution in Developing Countries: Micro Patterns of Turnover, Productivity, and Market Structure, New York, Oxford University Press.

Griliches, Zvi (1998) REDD and Productivity: the Econometric Evidence, University Of Chicago Press, Chicago.

Grossman, Sanford J., and Oliver D. Hart (1986), "The Costs and Benefits of Ownership: A Theory of Vertical and Lateral Integration," Journal of Political Economy, 94:4, pp. 691-719.

Grossman, Gene and Elhanan Helpman (1991), Innovation and Growth in the Global Economy, Cambridge, MA, MIT Press.

Hall, Robert and Charles I. Jones (1999), "Why Do Some Countries Produce So Much More Output per Worker Than Others?," Quarterly Journal of Economics, 114:1, pp. 83-116.

Hart, Oliver and John Moore (1990), "Property Rights and the Nature of the Firm," Journal of Political Economy, 98:6, pp. 1119-1158.

Holmström, Bengt (1982), "Moral Hazard in Teams," Bell Journal of Economics, 13, pp. 324-40.

Holmström, Bengt and Paul Milgrom (1991), "Multiactivity Principal-Agent Analyses: Incentive Contracts, Asset Ownership, and Job Design" Journal of Law, Economics and Organization 7, n0 (Special Issue): 24-52. 
Hopenhayn, Hugo A. (1992), "Entry, Exit, and firm Dynamics in Long Run Equilibrium," Econometrica, 60:5, pp. 1127-1150.

Klein, Benjamin, Crawford, Robert G., and Armen A. Alchian (1978), "Vertical Integration, Appropriable Rents, and the Competitive Contracting Process," Journal of Law and Economics, 21:2, pp. 297-326.

Klenow, Peter J. and Andres Rodriquez-Clare (1997), "The Neoclassical revival in Growth Economics: Has It Gone Too Far?," NBER Macroeconomics Annual, pp. 73-103.

Klette Tor Jacob (1996), "R\&D, Scope Economies, and Plant Performance," Rand Journal of Economics, 27, pp. 502-22.

Klette Tor Jacob and Samuel Kortum (2004), "Innovating Firms and Aggregate Innovation," Journal of Political Economy, 112, pp. 986-1018.

Levchenko, Andrei (2003), "Institutional Quality and International Trade," MIT Ph.D. dissertation.

Maskin, Eric and Jean Tirole (1999) "Unforeseen Contingencies and Incomplete Contracts," Review of Economic Studies, 66, pp. 83-114.

Martimort David and Thierry Verdier (2000), "The Internal Organization of the Firm, Transaction Costs and Macroeconomic Growth," Journal of Economic Growth, 5, pp. 315-40.

Martimort David and Thierry Verdier (2004), "The Agency Cost of Internal Collusion and Schumpeterian Growth," Review of Economic Studies, 71, pp. 1119-41.

Melitz, Marc J. (2003), "The Impact of Trade on Intra-Industry Reallocations and Aggregate Industry Productivity," Econometrica, 71:6, pp. 1695-1725.

Morrison, Catherine J. (1992), "Unraveling the Productivity Growth Slowdown in the United States, Canada and Japan: The Effects of Subequilibrium, Scale Economies and Markups," Review of Economics and Statistics LXXIV:3, pp. 381-393.

Nunn, Nathan (2005) "Relationship Specificity, Incomplete Contracts and the Pattern of Trade," Quarterly Journal of Economics (forthcoming).

Osborne, Martin and Ariel Rubinstein (1994), A Course in Game Theory, MIT Press, Cambridge, MA.

Quintin, Erwan (2003), "Limited Enforcement and the Organization of Production," mimeo.

Roberts, Mark J. (1996), "Colombia, 1977-85: Producer Turnover, Margins, and Trade Exposure," in Mark J. Roberts and James R. Tybout (eds.) (1996) Industrial Evolution in Developing Countries: Micro Patterns of Turnover, Productivity, and Market Structure, New York, Oxford University Press. 
Romer, Paul M. (1990), "Endogenous Technological Change," Journal of Political Economy, IIC, S71-S102.

Rotemberg, Julio J. and Michael Woodford (1991), "Markups and the Business Cycle," in Olivier Jean Blanchard and Stanley Fischer (eds.), NBER Macroeconomics Annual 1991, Cambridge, MA, The MIT Press.

Shapley, Lloyd S. (1953), "A Value for N-Person Games," in Contributions to the Theory of Games, pp. 31-40, Princeton University Press, Princeton, NJ.

Stole, Lars A. and Jeffrey Zwiebel (1996a), "Organizational Design and Technology Choice under Intrafirm Bargaining," American Economic Review, 86:1, pp. 195-222.

Stole, Lars A. and Jeffrey Zwiebel (1996b), "Intra-Firm Bargaining under Non-Binding Contracts," Review of Economic Studies, 63:3, pp. 375-410.

Sutton, John (1988), Technology and Market Structure: Theory and History, MIT Press, Massachusetts.

Tybout, James R. (2000), "Manufacturing Firms in Developing Countries: How Well Do They Do, and Why?," Journal of Economic Literature, 38:1, pp. 11-44

Williamson, Oliver E. (1975), Markets, Hierarchies: Analysis, Antitrust Implications, Free Press: New York.

Williamson, Oliver E. (1985), The Economic Institutions of Capitalism, Free Press: New York.

Yang, Xiaokai and Jeff Borland (1991), "A Microeconomic Mechanism for Economic Growth," Journal of Political Economy, 99:3, pp. 460-482. 